
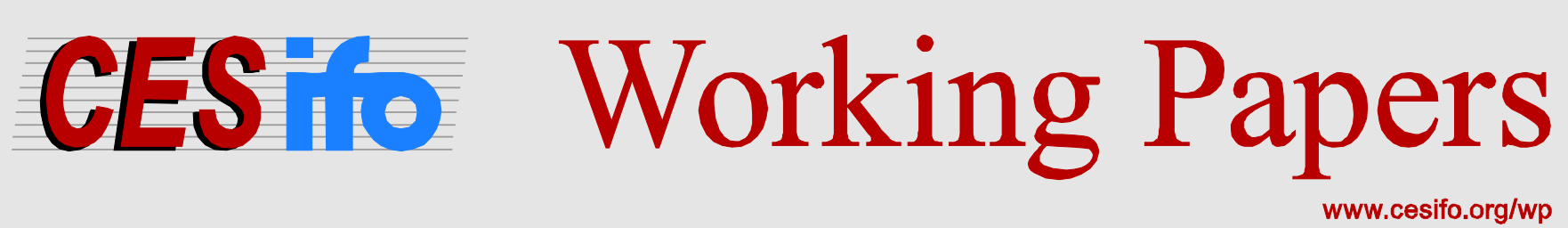

\title{
Delegation and Dynamic Incentives
}

\author{
Dongsoo Shin \\ Roland Strausz
}

CESIFO WORKING PAPER NO. 4774

CATEGORY 11: INDUSTRIAL ORGANISATION

APRIL 2014

An electronic version of the paper may be downloaded

- from the SSRN website:

- from the RePEc website:

- from the CESifo website:

WWW.SSRN.com

www.RePEc.org

www.CESifo-group.org/wp

\section{CESifo}




\title{
Delegation and Dynamic Incentives
}

\begin{abstract}
Using an agency model, we show how delegation, by generating additional private information, improves dynamic incentives under limited commitment. It circumvents ratchet effects and facilitates the revelation of persistent private information through two effects: a play-hardball effect, which mitigates an efficient agent's ratchet incentive, and a carrot effect which reduces an inefficient agent's take-the-money-and-run incentive. Although delegation entails a loss of control, it is optimal when uncertainty about operational efficiency is large. Moreover, delegation is more effective with production complementarity. We also consider different modes of commitment to yield insights into optimal organizational boundaries.
\end{abstract}

JEL-Code: D820, D860, L220.

Keywords: agency, delegation, dynamic incentives, limited commitment.

Dongsoo Shin

Department of Economics

Leavey School of Business

Santa Clara University

USA - Santa Clara, CA 95053

dshin@scu.edu
Roland Strausz

Institute for Microeconomic Theory

Humboldt University Berlin

Spandauer Str. 1

Germany-10178 Berlin

strauszr@wiwi.hu-berlin.de

The earlier version of this paper was circulated under the title "Hierarchical Structures and Dynamic Incentives”. We thank Helmut Bester, Wouter Dessein, Fahad Khalil, Daniel Krähmer, Jacques Lawarrée, Dilip Mookherjee, Martin Peitz, Michael Riordan and the seminar participants at Columbia University, University of British Columbia, University of Washington, University of California-Irvine, Indiana University Purdue University at Indianapolis, and Econometric Society Asian Meeting (2011) for their comments. We acknowledge financial supports from the Leavey Research Grant and the Deutsche Foschungsgemeinschaft (SFB-TR 15). 


\section{Introduction}

In dynamic environments a timely revelation of useful information is essential for organizations to implement efficient decisions. An organization, however, is susceptible to a dynamic incentive problem when its commitment power is limited. The ratchet effect, for example, is a well known problem - an organization's subdivisions or suppliers resist early revelation of persistent private information because they fear that the revealed information is used for future exploitation. As dynamic incentives play a crucial role for early information revelation, designing optimal structures for operations is a central issue in motivating the agents in a time consistent manner for organizations under limited commitment.

Using an agency framework, we present a theory of delegation based on the idea that delegation mitigates dynamic incentive problems that organizations face over time. In particular, we demonstrate that, by delegating additional tasks to its agents, an organization can increase the rate at which persistent private information is revealed - our analysis uncovers the mechanism by which delegation facilitates early information revelation and identifies the conditions under which delegation benefits the organization.

The question of the scope of delegation is central to a firm's operational structure and some highly successful firms heavily depend on delegated operational structures. Companies, such as British Petroleum, Johnson \& Johnson, and ABB, are well known for delegating their operations to the individual subdivisions without much central oversight (See Roberts 2004). Companies like Apple and 3M take delegation even beyond their organizational boundaries. They hardly own any manufacturing facilities and, instead, delegate also their manufacturing functions to their suppliers. Apple's supplier in China, for instance, not only provides labor inputs for the final assembly of the final product but also manufactures the capital intensive components such as semiconductors, LCD panels and camera devices. Companies like Samsung Electronics, by contrast, operate in a highly centralized structure (See . Samsung's local supplier merely provides labor inputs for the final assembly, with virtually no control over capital inputs. ${ }^{1}$ As we argue below our theory sheds light on these operational differences — delegation vs. centralization — by stressing the importance of dynamic information revelation.

A primary advantage of operating under centralization is that the organization has more direct control, whereas under delegation, the organization has to relinquish control and put

\footnotetext{
${ }^{1}$ Apple's and Samsung's operational structure are discussed in Lashinsky (2012) and Michell (2010).
} 
faith in the hands of its subdivisions or suppliers. Using a principal-agent model with persistent private information, we show that delegation can nevertheless be advantageous over centralization because it can ease the tension between different dynamic incentives. In our model, production requires both the agent's labor input, whose efficiency is persistent and his private information, and a capital input. The operational structure can be either centralization or delegation - the principal can centralize the operation by directly controlling the capital input, or delegate the control to the agent.

The principal's commitment power is limited in that she can commit to the operational structure, but cannot commit to the long-term output level and transfer to the agent. In the earlier period, the capital input uses a standard technology whose efficiency is commonly known. In the later period, due to technological progress, the efficiency of the capital input increases with some probability, and the party who is in charge of the input (determined by centralization or delegation) learns the efficiency privately.

In this framework, we identify a tension between two dynamic incentives, each of which is associated with a distinct but persistent labor efficiency of the agent - the ratchet incentive when the agent is efficient, and the take-the-money-and-run ${ }^{2}$ incentive when the agent is inefficient. An efficient agent is reluctant to reveal his efficiency in the earlier period, because he fears exploitation and forfeited rents of the persistent information in the later period. To avoid this, the organization must pay the future rents to the efficient agent as a large transfer in the earlier period. However, such a large transfer paid up-front to the efficient agent leads to another incentive problem. When the agent is inefficient, it raises the take-the-moneyand-run incentive in that the inefficient agent will misrepresent his labor efficiency in order to take the large transfer that is meant for the efficient agent in the earlier period, and subsequently reject the offer in the later period. The tension between these two incentives obstructs early information revelation under centralization.

In our model, two effects from delegating the control of the capital input to the agent ease the tension between the two dynamic incentives: the well-known play-hardball effect which relaxes the efficient agent's ratchet incentive, and the carrot effect which mitigates the inefficient agent's take-the-money-and-run incentive.

The play-hardball effect is well exploited in the literature. ${ }^{3}$ It mitigates the efficient

\footnotetext{
${ }^{2}$ This terminology is from Laffont and Tirole (1993) and Bolton and Dewatripont (2005).

${ }^{3}$ This effect, also known as termination effect, arises also in other dynamic environments (e.g., Bolton and Scharstein 1990 and Hirao 1993), but its beneficial role in mitigating ratchet effects has not been emphasized.
} 
agent's ratchet incentive by cutting output more credibly in the later period - these production cuts follow from increased costs of operation due to the agent's additional private information. The anticipated output cuts imply that the efficient agent's expected future rents from his private information become smaller. This, in turn, reduces the agent's opportunity cost of revealing his persistent information in the earlier period. In other words, the play-hardball effect reduces ratcheting and discourages the efficient agent from misrepresenting his efficiency in the earlier period.

In contrast, the carrot effect allows the organization to pledge rents to the agent in the later period, because under delegation, the agent anticipates a future rent from his additional private information. Therefore, if the inefficient agent decides to "run" after mimicking the efficient agent in the earlier period, he will lose this rent in the later period. Delegation therefore discourages the inefficient agent from exaggerating his labor efficiency in the earlier period to play the take-the-money-and-run strategy.

According to our analysis, when the organization faces small uncertainty about operational efficiency, only one of the two effects obtains - the play-hardball effect when operation is likely to be efficient, and the carrot effect when the operation is likely to be inefficient. In such cases, delegation is optimal only if the organization's capacity expands by a small amount over time. When the uncertainty about operational efficiency is large, however, both the carrot and the play-hardball effect arise and reinforce each other. In such a case, delegation is optimal regardless of the organization's capacity expansion.

Our result is consistent with some empirical studies that address the issue of delegation in operations. For example, Acemoglu et al. (2007) demonstrate that organizations with less information tend to choose decentralized structures for their operations. Specifically, they show that firms closer to the technological frontier, firms in more heterogenous environments, and younger firms are more likely to operate under delegation. These empirical observations are in line with our theoretical prediction that uncertainty favors delegation. ${ }^{4}$

Our findings are also in agreement with organization studies such as Mintzberg (1979) who argues that centralized structures tend to be adopted when the organization operates in a relatively stable and predictable environment, whereas decentralized structures tend to be adopted when the organization faces a less stable and unpredictable environment. Also Cyert and March (1963/1992) note that having the right information in earlier stages of

\footnotetext{
${ }^{4}$ We note however that other empirical studies (e.g., Kastl et al. 2013) do not find a significant relationship between a firm's age and its operational structure.
} 
operation is one of the most important factors for an organization when it faces a large uncertainty. Our result suggests that delegation serves organizations for such a purpose.

This article also sheds light on differences in operating structures of industry leaders and followers. Our result suggests that industry leaders, facing larger uncertainties, have a tendency to delegate more, whereas industry followers tend to operate in a more centralized way. For example, in consumer electronics industry, a company like Apple is an industry leader that operates under a great deal of uncertainties. As mentioned above, the company delegates the entire manufacturing activities to its supplier. By contrast, an industry follower such as Samsung Electronics directly controls its own capital intensive components. ${ }^{5}$

We extend our analysis to the following two directions. First, we show that complementarity in operations tend to favor delegation. In particular, the play-hardball effect of delegation is linked to complementarity in production, whereas the carrot effect can survive even when the inputs are perfect substitutes.

Second, we demonstrate that our results are robust when centralization allows for more commitment than delegation — we allow the organization under centralization to offer a long-term contract, but with a possibility of renegotiation. ${ }^{6}$ Following the distinction in Williamson (1985) that vertical integration enables organizations to use long-term contracts, whereas vertically separated inter-firm transactions are often restricted to short-term contracts, this extension allows us to apply our results to the issue of organizational boundary.

The rest of the article is organized as follows. The related literature is discussed in Section 2. We present the model in Section 3. Centralization under which the principal directly controls the capital input is analyzed in Section 4. Delegation of the control to the agent is analyzed in Section 5. Extensions of our analysis are discussed in Section 6. Section 7 concludes. All proofs are relegated to Appendix A. In Appendix B, we demonstrate that our results are robust when considering semi-separating strategies.

\section{Related Literature}

There are previous studies that provide economic rationales for delegation in dynamic environments, but they do not examine its role in facilitating dynamic information revelation in

\footnotetext{
${ }^{5}$ In an interview with the New York Times, (February 13, 2013), Samsung's executive vice president adhered to this view of Apple as the industry leader and Samsung as a follower. Michell (2010) describes Samsung's centralized operation as an "obsession with owning a factory."

${ }^{6}$ We thank the editor for suggesting to consider this extension.
} 
the presence of ratchet effects. Crémer (1995), using an incomplete contract approach, shows that an organization may benefit from delegation that limit information flows and keep the agent at a distance. Beaudry and Poitevin (1995) point out that delegation can make it harder to achieve a successful renegotiation. ${ }^{7}$ Aghion and Tirole (1997) demonstrate that delegation induces acquisition of useful information for the organization. In a model with full commitment, Laffont and Martimort (1998) show that delegation enables the organization to effectively discriminate transfers among different agents, thus mitigating the agents' incentives to collude. ${ }^{8}$ Studies such as Dessein (2002) and Alonso et al. (2008) show that organizations can benefit from delegation because it makes better use of private information.

The following studies exploit the links between operational structures and dynamic incentives. Meyer et al. (1996) show that assigning the agents joint responsibility for tasks alleviates ratchet problems. Olsen and Torsvik (2000) show that a firm's ability to learn about the difficulty of the tasks workers engage in will induce the firm to give workers more discretion over tasks and weaker incentives. ${ }^{9}$ Aghion et al. (2004) shows that delegation of the earlier task induces the worker to reveal his private information, of which the project owner can take advantage in making a decision for the later task. None of them, however, consider a tension aroused from different incentives of the agent of different types.

Finally, there are studies related to the extensions in our article. Dewatripont and Maskin (1995), for example, show that organizations may want to deliberately limit the contingencies on which an incentive contract should depend when renegotiation is possible. Unlike their study, our focus is on operational structures and organizational boundaries. Olsen (1996) shows that, under renegotiation, vertical integration can be dominated by vertical separation. In his study, however, vertical separation is not accompanied by increased proportion of manipulable information and decreased explicit commitment power. In our study, vertically separated transactions can only be governed by short-term contracts, and induce more private information on the agent's part. In this sense, our finding complements the author's study. Severinov (2008) shows that the optimal structure of operation depends on the production technology. In his study, however, delegation is weakly dominated regardless of complementarity or substitutability of the inputs.

\footnotetext{
${ }^{7}$ Poitevin (2000) extends their analysis to a multi-agent setting.

${ }^{8}$ The authors consider contractual delegation in their paper. See also Baron and Besanko (1992), Rajan and Zingales (2001) and Dequiedt and Martimort (2004) for studies on contractual delegation.

${ }^{9}$ In Olsen and Torsvik $(1993,1995)$, the authors address dynamic incentive problems in common agency. See also Martimort (1999) who analyze a similar situation.
} 


\section{The Model}

We study a dynamic adverse selection model with persistent private information, in which a principal hires an agent for the production over two periods $(t=1,2)$. Production requires two complementary inputs: labor and capital. The labor input must be provided by the agent. The agent's labor efficiency, $\theta^{L}$, is efficient, $\theta^{h}$, with probability $\mu$, and inefficient, $\theta^{l}$, with probability $1-\mu$, where $0<\theta^{l}<\theta^{h}<1$. The agent's labor efficiency is his private information and persistent in that it does not change between the two periods.

Unlike the labor input, the control of the capital input can be allocated to either the principal or the agent. We denote by $\Psi \in\{C, D\}$ the firm's operational structure regarding the allocation of control. Under centralization, $\Psi=C$, the principal directly controls the capital input, whereas under delegation, $\Psi=D$, the agent controls it. The party who controls the capital input bears the cost of the input. To make our point in a parsimonious setting, we assume that the capital input in $t=1$ uses a standard technology whose efficiency is commonly known. In $t=2$, the capital efficiency improves due to technological progress. In line with Riordan (1990), we assume that only the party who controls it privately learns its efficiency.

The First Period In the first period $(t=1)$, the labor and the capital input levels, denoted by $q_{\lambda 1}$ and $q_{\kappa 1}$ respectively, are: $q_{\lambda 1}, q_{\kappa 1} \in\{0,1\} .{ }^{10}$ The marginal cost of the agent's labor input is given by $1-\theta^{L}$, where $L \in\{h, l\}$. As mentioned above, the capital efficiency in $t=1$ is common knowledge. The marginal cost of the capital input is normalized to 1 . Because the labor and the capital input are complements, they yield an output $Q_{1}=2 \min \left\{q_{\lambda 1}, q_{\kappa 1}\right\}$. Therefore $q_{\lambda 1}=q_{\kappa 1}$ in equilibrium, and we let:

$$
q_{1}=q_{\lambda 1}=q_{\kappa 1} \in\{0,1\}
$$

Hence, $Q_{1}=2 q_{1}$, the cost of the labor input is $\left(1-\theta^{L}\right) q_{1}, L \in\{h, l\}$, and the cost of the capital input is $q_{1}$ in $t=1$. The principal pays a transfer $T_{1}$ to the agent in $t=1$.

The Second Period In the second period $(t=2)$, technological progress affects efficiency of the capital input and production capacity. The capital efficiency in $t=2$ is denoted by $\theta^{K}$, which measures the size of the reduction in marginal cost from 1 to $1-\theta^{K}$. Again,

\footnotetext{
${ }^{10}$ We use a setup with binary inputs for tractability. As is well known (e.g., Laffont and Tirole 1988 or Drugov 2010), dynamic adverse selection models with limited commitment become quickly intractable.
} 
$\theta^{K}$ is privately observed by the party who controls it before the second period contract is offered. It is efficient, $\theta^{h}$, with probability $\mu$ and inefficient, $\theta^{l}$, with probability $1-\mu{ }^{11}$ The efficiency of the capital and the labor input are stochastically independent. Production capacity increases in $t=2$, and as a result, the labor and the capital input levels, denoted by $q_{\lambda 2}$ and $q_{\kappa 2}$ respectively, are: $q_{\lambda 2}, q_{\kappa 2} \in\{0, \gamma\}$, where $\gamma>1$. As before, $Q_{2}=2 \min \left\{q_{\lambda 2}\right.$, $\left.q_{\kappa 2}\right\}$, and therefore $q_{\lambda 2}=q_{\kappa 2}$ in equilibrium. We again let:

$$
q_{2}=q_{\lambda 2}=q_{\kappa 2} \in\{0, \gamma\}
$$

Hence, $Q_{2}=2 q_{2}$ and input costs are $\left(1-\theta^{L}\right) q_{2}$ and $\left(1-\theta^{K}\right) q_{2}, L, K \in\{h, l\}$ in $t=2$. The principal pays the transfer $T_{2}$ to the agent in $t=2$.

Limited Commitment The principal can commit to the long-term operational structure, $\Psi \in\{C, D\}$, but not to the long-term output levels and transfers - the output targets and transfers are decided and offered to the agent in the beginning of each period. This assumption captures limits on commitment in practise. ${ }^{12}$ As well documented, even when the top management of an organization has a clear vision of a new strategy, managing operational change is extremely difficult or impossible (see Argyris, 1982 and Schein, 2004), ${ }^{13}$ whereas wages and production targets are often reneged. The organization's limited commitment implies the following structure and timing of contracting. At the outset, the principal commits to the operational structure $\Psi \in\{C, D\}$. In the beginning of $t=1$, the principal offers the agent the first period contract that specifies the input level $q_{1}\left(=q_{\lambda 1}=q_{\kappa 1}\right)$ and the transfer to the agent $T_{1}$. The transfer compensates the agent for his labor input and, depending on the operational structure, the cost of the capital input. In the beginning of $t=2$, the principal's offer specifies the input level $q_{2}\left(=q_{\lambda 2}=q_{\kappa 2}\right)$ and the transfer to the agent $T_{2}$. The offer in $t=2$ must be sequentially rational - it must be optimal in the beginning of $t=2$, conditional on the entire information that the principal has learned up to that point.

Our approach is to analyze first the implementable outcomes under centralization and

\footnotetext{
${ }^{11}$ For simplicity, we consider the symmetric case, $\mu=\mu_{\lambda}=\mu_{\kappa}, \theta^{l}=\theta_{\lambda}^{l}=\theta_{\kappa}^{l}$ and $\theta^{h}=\theta_{\lambda}^{h}=\theta_{\kappa}^{h}$. Later, we extend our study to the case with input specific cost differences. The case where $\mu_{\lambda} \neq \mu_{\kappa}$ is presented in the earlier version of this paper (accessible at http://www4.gsb.columbia.edu/filemgr?file_id=7310604).

${ }^{12}$ The limited commitment in our paper is a standard assumption in the literature. See also Dessein (2002) and Alonso et al. (2008) for similar assumptions.

${ }^{13}$ See also Economist (July 20th 2000) reporting that bad performances of many mergers are due to unreconcilable difference in operational structures between the merging firms that failed to be harmonized.
} 
delegation with a special regard to the timing of information revelation. We then compare their optimality from the principal's perspective. We start our analysis with centralization.

\section{Centralization $(\Psi=C)$}

An obvious advantage of centralization is that the principal has direct control over the capital input and thus learns its efficiency. As a result, she does not have to provide any incentives for eliciting the information on the capital efficiency from the agent. On the other hand, a possible complication is that, because $\theta^{K}$ is the principal's private information, the agent may interpret the principal's contract offer in the second period as a signal about her private information. Under centralization, however, the capital efficiency $\theta^{K}$ is irrelevant to the agent, and thus such signaling is inconsequential. This irrelevance actually allows us to simplify the analysis by treating the setup under centralization as if the second period contract can condition directly on the true $\theta^{K} .{ }^{14}$ With this in mind, we can now describe the contracting game that results in the centralized operational structure $\Psi=C$.

The first period contract specifies the input level $q_{1}$ and the transfer $T_{1}$. Under centralization, the principal incurs the cost of the capital input, which is $q_{1}$ in $t=1$. The principal's and the agent's first period payoffs are respectively:

$$
\Pi_{1}^{C}\left(q_{1}, T_{1}\right) \equiv 2 q_{1}-T_{1}-q_{1} \quad \text { and } \quad U_{1}^{C}\left(q_{1}, T_{1} \mid \theta^{L}\right) \equiv T_{1}-\left(1-\theta^{L}\right) q_{1} .
$$

Because the capital efficiency in $t=1$ is commonly known, the first period contract $\Phi_{1}^{C}$ is contingent only on the agent's report on his labor efficiency $\theta^{L}$. The first period contract under centralization has the structure:

$$
\Phi_{1}^{C}=\left\{q_{1}\left(\theta_{1}^{L}\right), T_{1}\left(\theta_{1}^{L}\right)\right\}, \quad L \in\{h, l\}
$$

where $\theta_{1}^{L}$ is the agent's report on $\theta^{L}$ in $t=1$. For notational convenience, we let $q_{1}^{L} \equiv q_{1}\left(\theta_{1}^{L}\right)$ and $T_{1}^{L} \equiv T\left(\theta_{1}^{L}\right)$, where $L \in\{h, l\}$.

Similarly, the second period contact, specifying the input level $q_{2}$ and the transfer $T_{2}$, results in the following payoffs for the principal and the agent respectively:

$$
\Pi_{2}^{C}\left(q_{2}, T_{2} \mid \theta^{K}\right) \equiv 2 q_{2}-T_{2}-\left(1-\theta^{K}\right) q_{2} \quad \text { and } \quad U_{2}^{C}\left(q_{2}, T_{2} \mid \theta^{L}\right) \equiv T_{2}-\left(1-\theta^{L}\right) q_{2} .
$$

\footnotetext{
${ }^{14}$ It is indeed straightforward to check that all equilibrium outcomes that we obtain depend on $\theta^{K}$ in such a way that the principal does not have an incentive to misrepresent $\theta^{K}$. Technically speaking, we have an informed principal problem, which is innocuous because it concerns only private values.
} 
Under centralization the principal learns $\theta^{K}$ privately, but as explained above, we can treat the information as public. Because, after $t=1$ the agent's labor efficiency $\theta^{L}$ may or may not be revealed, we express the second period offer, $\Phi_{2}^{C}$, as:

$$
\Phi_{2}^{C}=\left\{q_{2}\left(\theta_{2}^{L}, \theta^{K}\right), T_{2}\left(\theta_{2}^{L}, \theta^{K}\right)\right\}, \quad L, K \in\{h, l\}
$$

where $\theta_{2}^{L}$ is the agent's report in $t=2$ about his labor efficiency $\theta^{L}$. For notational convenience, we let $q_{2}^{L K} \equiv q_{2}\left(\theta_{2}^{L}, \theta^{K}\right)$ and $T_{2}^{L K} \equiv T\left(\theta_{2}^{L}, \theta^{K}\right)$, where $L, K \in\{h, l\}$.

The timing of the contracting game under centralization $\Psi=C$ is as follows:

1.1 The principal offers a first period contract $\Phi_{1}^{C}$ to the agent, who accepts/rejects.

1.2 If the offer is accepted, the agent sends a report $\theta_{1}^{L}$.

1.3 Contract $\Phi_{1}^{C}$ is executed and period 1 ends with the corresponding payoffs.

2.0 The capital efficiency gets improved and $\theta^{K}$ is publicly revealed.

2.1 The principal offers a second period contract $\Phi_{2}^{C}$ to the agent, who accepts/rejects.

2.2 If the offer is accepted, the agent sends a report $\theta_{2}^{L}$.

2.3 Contract $\Phi_{2}^{C}$ is executed and players receive their corresponding second period payoffs.

For this timing, we analyze the principal's optimal offers. Because of the principal's limited commitment, the question will not only be how the principal induces a revelation of private information optimally, but also when any such revelation is to occur. More specifically, the question is whether it is optimal for the principal to structure contracts such that the first period report $\theta_{1}^{L}$ reveals already all the information about $\theta^{L}$, or whether it is better to use also the second report $\theta_{2}^{L}$ for revealing information about $\theta^{L}$.

To address the timing of information revelation, we classify first period contracts $\Phi_{1}^{C}$ by the degree of early information revelation they induce. For expositional reasons, we will concentrate on the two extremes: first period contracts that induce no information revelation and those that induce full information revelation. We relegate the formal analysis of the intermediate case to Appendix B, where we demonstrate that delegation is optimal also with respect to a first period contract that induces a partial revelation of information (semi-separating). 


\section{Centralization without Early Information Revelation}

A first period contract that does not elicit any direct or indirect information from the agent induces the same outcome in $t=1$ regardless of the agent's labor efficiency. Such a contract is equivalent to a first period contract that is independent of the agent's report on $\theta^{L}$. Therefore, we can express such a pooling contract for $t=1$ as follows:

$$
\Phi_{1}^{C P}=\left\{q_{1}, T_{1}\right\}
$$

With a pooling contract, no information is revealed in $t=1$. Hence, in the beginning of $t=2$, the principal still has the prior belief $\mu$ that the agent's labor input is efficient. Because the principal learns $\theta^{K}$ directly under centralization, in $t=2$ the sequentially rational offer, $\Phi_{2}^{C P}=\left\{T_{2}^{h K}, q_{2}^{h K}, T_{2}^{l K}, q_{2}^{l K}\right\}$ for each case of $K \in\{h, l\}$, maximizes her expected payoff:

$$
\Pi_{2}^{C P}=\mu \Pi_{2}^{C}\left(q_{2}^{h K}, T_{2}^{h K} \mid \theta^{K}\right)+(1-\mu) \Pi_{2}^{C}\left(q_{2}^{l K}, T_{2}^{l K} \mid \theta^{K}\right),
$$

subject to the agent's participation constraints,

$$
U_{2}\left(q_{2}^{L K}, T_{2}^{L K} \mid \theta^{L}\right) \geq 0, \quad L \in\{h, l\}
$$

and the agent's incentive compatibility constraints,

$$
U_{2}\left(q_{2}^{L K}, T_{2}^{L K} \mid \theta^{L}\right) \geq U_{2}\left(q_{2}^{L^{\prime} K}, T_{2}^{L^{\prime} K} \mid \theta^{L}\right), \quad L, L^{\prime} \in\{h, l\}
$$

The principal's maximization problem reflects the familiar trade-off between rents and productive efficiency. In $t=2$, the principal's first option is to offer again a pooling contract that guarantees production but at the expense of an information rent to the efficient agent. Alternatively, she can save on information rents by offering a separating contract that only induces the efficient agent to engage in production. Because the principal's trade-off between these two options depends on her beliefs about the agent's labor efficiency, we introduce the following definition.

Definition 1 For $x \in[0,1]$, we let:

$$
\hat{\mu}(x) \equiv \frac{\theta^{l}+x}{\theta^{h}+x} .
$$

With this definition the following lemma characterizes the principal's sequentially rational contract in $t=2$. 
Lemma 1 Given $\Phi_{1}^{C P}$ in $t=1$, the sequentially rational contract $\Phi_{2}^{C P}$ in $t=2$ is as follows:

i) If $\mu \leq \hat{\mu}\left(\theta^{l}\right)$, then $q_{2}^{L K}=\gamma ; T_{2}^{L K}=\left(1-\theta^{l}\right) \gamma ; L, K \in\{h, l\}$.

ii) If $\mu \in\left(\hat{\mu}\left(\theta^{l}\right), \hat{\mu}\left(\theta^{h}\right)\right]$, then $q_{2}^{h h}=q_{2}^{h l}=q_{2}^{l h}=\gamma, q_{2}^{l l}=0 ; T_{2}^{h h}=T_{2}^{l h}=\left(1-\theta^{l}\right) \gamma$, $T_{2}^{h l}=\left(1-\theta^{h}\right) \gamma, T_{2}^{l l}=0$.

iii) If $\mu>\hat{\mu}\left(\theta^{h}\right)$, then $q_{2}^{h K}=\gamma, q_{2}^{l K}=0 ; T_{2}^{h K}=\left(1-\theta^{h}\right) \gamma, T_{2}^{l K}=0 ; K \in\{h, l\}$.

The lemma shows that, after offering a pooling contract in $t=1$, the principal also pools in $t=2$ when her belief about the agent's efficiency is relatively pessimistic. When pessimistic, the principal provides information rents for ensuring production by pooling the agent's efficiencies in $t=2$. In contrast, separation is optimal when the principal has optimistic beliefs about the agent's type. For intermediate beliefs, Lemma 1 shows that the principal's decision to pool or to separate in $t=2$ depends on the efficiency of the capital input. More specifically, the principal is more eager to have production when the capital input is efficient. The reason is that, when the capital input is efficient, production is more valuable so that the principal is more inclined to offer a pooling contract to ensure production.

With Lemma 1, the principal's optimal payoff from a pooling offer in $t=1$ follows straightforwardly.

Proposition 1 Under centralization, the principal's maximum expected payoff without inducing early information revelation is:

$$
\Pi^{C P}= \begin{cases}\theta^{l}+\left(\theta^{l}+\widetilde{\theta}\right) \gamma & \text { if } \mu \leq \hat{\mu}\left(\theta^{l}\right), \\ \theta^{l}+\mu(2-\mu)\left(\theta^{h}+\theta^{l}\right) \gamma & \text { if } \mu \in\left(\hat{\mu}\left(\theta^{l}\right), \hat{\mu}\left(\theta^{h}\right)\right] \\ \theta^{l}+\mu\left(\theta^{h}+\widetilde{\theta}\right) \gamma & \text { if } \mu>\hat{\mu}\left(\theta^{h}\right) .\end{cases}
$$

\section{Centralization with Early Information Revelation}

A first period contract that induces early information revelation leads to separating the agent's labor efficiencies in $t=1$. We denote such a separating contract as $\Phi_{1}^{C S}$, which induces the agent to reveal his labor efficiency completely in $t=1$. Thus, a first period separating contract $\Phi_{1}^{C S}$ has a structure as in (1) and, in addition, satisfies incentive constraints which ensure the agent's truthful report on $\theta^{L}$. 
With a first period separating contract, the principal learns the agent's labor efficiency in $t=1$. In $t=2$, therefore, centralization allows the principal to make her contract offer under full information. It is therefore straightforward to derive the sequentially rational contract offer in $t=2$.

Lemma 2 Given $\Phi_{1}^{C S}$ in $t=1$, the sequentially rational contract $\Phi_{2}^{C S}$ in $t=2$ is: $q_{2}^{L K}=\gamma$; $T_{2}^{L K}=\left(1-\theta^{L}\right) \gamma ; L, K \in\{h, l\}$.

The lemma above shows that in $t=2$, the agent is fully exploited, i.e., receives zero rent regardless of his labor efficiency. It follows that the lemma captures the potential "ratchet" incentive due to the principal's limited commitment. Without any pre-commitment, the principal in $t=2$ offers the contract, taking into account all information that she has learned and, in particular, the agent's revelation of his efficiency in $t=1$.

Using Lemma 2, we are now ready to discuss the first period contract that separates the agent's labor efficiencies $\left(q_{1}^{h} \neq q_{1}^{l}\right)$. As usual, incentive compatibility of the first period contract $\Phi_{1}^{C S}$ implies a monotone production schedule $q_{1}^{h} \geq q_{1}^{l}$ so that separation requires $q_{1}^{h}=1$ and $q_{1}^{l}=0$. Next, we examine if the principal's offer can in fact separate the agent's labor efficiencies in $t=1$.

Lemma 3 Under centralization, a separating contract in $t=1$ cannot be implemented.

The intuition behind the lemma is as follows. As explained above, the efficient agent will be exploited in $t=2$ if he reveals his true efficiency in $t=1$. This is fully anticipated by the agent, and therefore the sum of rents over the two periods must be paid up-front for the efficient agent for an information revelation in $t=1$. Such a generous first period offer is, however, also attractive to the inefficient agent and induces him to play the "take-the-moneyand-run" strategy. That is, the inefficient agent will take the principal's offer in $t=1$ (for the efficient agent) by misrepresenting his efficiency, but he will not take the offer in $t=2$ because his rent in that period will be negative if he takes the offer. The infeasibility of early information revelation is therefore due to a combination of two problems: the rachet problem associated with the efficient agent, and the take-the-money-and-run problem associated with the inefficient agent.

Lemma 3 further implies that the payoff, $\Pi^{C P}$, as presented in Proposition 1 represents the principal's maximum payoff under centralization. 


\section{Delegation $(\Psi=D)$}

We next analyze the structure of optimal contracts under delegation $(\Psi=D)$, where the agent is in charge of the capital input so that he privately learns its efficiency in $t=2$. As in the case of centralization, the first period contract under delegation, $\Phi_{1}^{D}$, specifies the input level $q_{1}$ and the transfer $T_{1}$. Under delegation the agent incurs the cost of the capital input. Hence, the principal's and the agent's first period payoffs are respectively:

$$
\Pi_{1}^{D}\left(q_{1}, T_{1}\right) \equiv 2 q_{1}-T_{1} \quad \text { and } \quad U_{1}^{D}\left(q_{1}, T_{1} \mid \theta^{L}\right) \equiv T_{1}-\left(1-\theta^{L}\right) q_{1}-q_{1}
$$

Likewise, the principal's and the agent's second period payoffs are respectively:

$$
\Pi_{2}^{D}\left(q_{2}, T_{2}\right) \equiv 2 q_{2}-T_{2} \quad \text { and } \quad U_{2}^{D}\left(q_{2}, T_{2} \mid \theta^{L}, \theta^{K}\right) \equiv T_{2}-\left(1-\theta^{L}\right) q_{2}-\left(1-\theta^{K}\right) q_{2}
$$

Because the capital efficiency in the first period is common knowledge, $\Phi_{1}^{D}$ has the same structure as the one under centralization. The second period contract $\Phi_{2}^{D}$, however, is contingent on the agent's report on both $\theta^{L}$ and $\theta^{K}$ because both the labor and the capital efficiency are the agent's private information. We express the second period contract as:

$$
\Phi_{2}^{D}=\left\{q_{2}\left(\theta_{2}^{L}, \theta_{2}^{K}\right), T_{2}\left(\theta_{2}^{L}, \theta_{2}^{K}\right)\right\}, \quad L, K \in\{h, l\}
$$

where $\theta_{2}^{L}$ and $\theta_{2}^{K}$ are the agent's reports on the efficiencies of the two inputs. As before, $\Phi_{2}^{D}$ must be sequentially rational given the first period contract, $\Phi_{1}^{D}$. Again for convenience, we let $q_{2}^{L K} \equiv q_{2}\left(\theta_{2}^{L}, \theta^{K}\right)$ and $T_{2}^{L K} \equiv T_{2}\left(\theta_{2}^{L}, \theta^{K}\right)$, where $L, K \in\{h, l\}$.

The timing of the contracting game under delegation $\Psi=D$ is as follows:

1.1 The principal offers a first period contract $\Phi_{1}^{D}$ to the agent, who accepts/rejects;

1.2 If the offer is accepted, the agent sends a report $\theta_{1}^{L}$;

1.3 Contract $\Phi_{1}^{D}$ is executed and period 1 ends with the corresponding payoffs.

2.0 The capital efficiency gets improved and $\theta^{K}$ is privately observed by the agent.

2.1 The principal offers a second period contract $\Phi_{2}^{D}$ to the agent, who accepts/rejects;

2.2 If the offer is accepted, the agent sends a report $\theta_{2}^{L}$ and a report $\theta_{2}^{K}$;

2.3 Contract $\Phi_{2}^{D}$ is executed and players receive their corresponding second period payoffs. 
As before, we distinguish between first period pooling contracts $\Phi_{1}^{D P}$ which induce no early information revelation and separation contracts $\Phi_{1}^{D S}$ which induce full information revelation.

\section{Delegation without Early Information Revelation}

A first period contract that induces no early information revelation implies pooling in $t=1$ and is therefore expressed as:

$$
\Phi_{1}^{D P}=\left\{q_{1}, T_{1}\right\}
$$

Without information revelation in $t=1$, the agent in $t=2$ is privately informed of the efficiencies of both the labor and the capital input. From the principal's perspective, the situation in $t=2$ is equivalent to facing an agent with three possible cost types $\Theta^{h} \equiv 2 \theta^{h}$, $\Theta^{m} \equiv \theta^{h}+\theta^{l}$, and $\Theta^{l} \equiv 2 \theta^{l}$ with the respective probabilities $\varphi^{h} \equiv \mu^{2}, \varphi^{m} \equiv 2 \mu(1-\mu)$, and $\varphi^{l} \equiv(1-\mu)^{2}$. Therefore, without early information revelation, there is no trade-off between centralization and delegation, because delegation simply provides more private information to the agent compared to centralization. The following lemma shows this intuitive results formally.

Lemma 4 The principal's maximum expected payoff under delegation without early information revelation is strictly smaller than her maximum expected payoff under centralization.

As a result it is clear that, if the principal benefits from delegation at all, then the benefits arise from a possibility to induce early information revelation. We investigate this possibility next.

\section{Delegation with Early Information Revelation}

As in the case of centralization, we can express a first period contract with early information revelation as:

$$
\Phi_{1}^{D S}=\left\{q_{1}^{L}, T_{1}^{L}\right\}, \quad L \in\{h, l\}
$$

In addition, $\Phi_{1}^{D S}$ must be incentive compatible so that the agent truthfully reports $\theta^{L}$ in $t=1$. Again, a first period separating contract exhibits $q_{1}^{h}=1$ and $q_{1}^{l}=0$.

With separation in $t=1$, the principal receives an informative report about the agent's true labor efficiency in $t=1$. This report affects the principal's beliefs and hence her 
subsequent contract in $t=2$. Thus, we can view the principal's second period offer as contingent on the agent's report on his labor efficiency in $t=1$ and his report on the capital efficiency in $t=2$. The principal's second period contract is expressed as:

$$
\Phi_{2}^{D S}=\left\{q_{2}^{L K}, T_{2}^{L K}\right\}, \quad L, K \in\{h, l\}
$$

where superscript $L$ represents the agent's report $\theta_{1}^{L}$ about his labor efficiency in $t=1$ and superscript $K$ represents his report $\theta_{2}^{K}$ about the capital input efficiency in $t=2$.

With $\Phi_{1}^{D S}$, the principal will be fully informed about the agent's labor efficiency after the agent sends his report in $t=1$. Therefore, the sequentially rational contract for the second period, $\Phi_{2}^{D S}$ after a first period report $\theta_{1}^{L} \in\left\{\theta^{h}, \theta^{l}\right\}$ maximizes her second period payoff:

$$
\Pi_{2}^{D S} \equiv \mu \Pi_{2}^{D}\left(q_{2}^{L h}, T_{2}^{L h}\right)+(1-\mu) \Pi_{2}^{D}\left(q_{2}^{L l}, T_{2}^{L l}\right),
$$

subject to the agent's participation constraints,

$$
U_{2}^{D}\left(q_{2}^{L K}, T_{2}^{L K} \mid \theta^{L}, \theta^{K}\right) \geq 0, \quad K \in\{h, l\}
$$

and the incentive compatibility constraints,

$$
U_{2}^{D}\left(q_{2}^{L K}, T_{2}^{L K} \mid \theta^{L}, \theta^{K}\right) \geq U_{2}^{D}\left(q_{2}^{L K^{\prime}}, T_{2}^{L K^{\prime}} \mid \theta^{L}, \theta^{K}\right), \quad K, K^{\prime} \in\{h, l\} .
$$

Again, the principal faces a trade-off between pooling and separating - ensuring output vs. extracting information rent. With separation in $t=1$, however, the agent's private information in $t=2$ now concerns only the efficiency of the capital input, $\theta^{K}$. The next lemma shows how the principal's sequentially rational offer in $t=2$ depends on her belief that the capital input is efficient.

Lemma 5 Given $\Phi_{1}^{D S}$ in $t=1$, the sequentially rational contract $\Phi_{2}^{D S}$ in $t=2$ is:

i) If $\mu \leq \hat{\mu}\left(\theta^{l}\right)$, then $q_{2}^{L K}=\gamma ; T_{2}^{L K}=\left(2-\theta^{L}-\theta^{l}\right) \gamma, L, K \in\{h, l\} ;$ The agent gets a rent of $\Delta \theta \gamma$ in $t=2$ when $\theta^{K}=\theta^{h}$.

ii) If $\mu \in\left(\hat{\mu}\left(\theta^{l}\right), \hat{\mu}\left(\theta^{h}\right)\right]$, then $q_{2}^{h h}=q_{2}^{h l}=q_{2}^{l h}=\gamma, q_{2}^{l l}=0 ; T_{2}^{h h}=T_{2}^{h l}=T_{2}^{l h}=\left(2-\theta^{h}-\theta^{l}\right) \gamma$, $T_{2}^{l l}=0 ;$ The agent gets a rent of $\Delta \theta \gamma$ in $t=2$ when $\theta^{L}=\theta^{K}=\theta^{h}$.

iii) If $\mu>\hat{\mu}\left(\theta^{h}\right)$, then $q_{2}^{L h}=\gamma, q_{2}^{L l}=0 ; T_{2}^{L h}=\left(2-\theta^{L}-\theta^{h}\right) \gamma, T_{2}^{L l}=0 ; L \in\{h, l\}$; The agent gets no rent in $t=2$. 
Although Lemma 5 underlies a similar intuition as before, it is important to note that the optimal sequential rational contract now depends on the principal's belief about the capital input efficiency rather than the agent's labor efficiency. Moreover, the cutoff point of $\mu$ at which pooling prevails over separation depends on the agent's report in $t=1$, because it determines the principal's cost of foregone revenues from abandoning output in $t=2$.

Crucial for our subsequent analysis is the agent's rent from the sequentially rational contract, $\Phi_{2}^{D S}$, because it determines to what extent the principal can induce the agent to reveal his information in $t=1$. Importantly, not only the agent's rent on the equilibrium matters, but also his potential rent off the equilibrium. Thus, we need to examine explicitly the agent's strategy for the "off-the-equilibrium" event that the agent with labor efficiency $\theta^{L}$ misreports his efficiency by sending a report $\left(\theta_{1}^{L} \neq \theta^{L}\right)$ in $t=1$, and subsequently faces the sequentially rational contract $\Phi_{2}^{D S}$ in $t=2$.

Lemma 6 If the inefficient agent misreported his type as efficient in $t=1$, then he will reject $\Phi_{2}^{D S}$ in $t=2$. If the efficient agent misreported his type as inefficient in $t=1$, then he will still accept $\Phi_{2}^{D S}$ in $t=2$ and report the capital efficiency $\theta^{K}$ truthfully.

Lemma 6 confirms that, like under centralization, the inefficient agent will, in case of misreporting in $t=1$, adopt the take-the-money-and-run strategy by rejecting the principal's offer for $t=2$. For the efficient agent, the sequentially rational contract in $t=2$ is individually rational and incentive compatible irrespective of his report in $t=1$. Then, parallel to Lemma 3 , the question is whether there exists a contract $\Phi_{1}^{D S}$ that keeps the agent from misreporting in $t=1$.

Lemma 7 A separating contract $\Phi_{1}^{D S}$ exists when one of the following conditions holds:

i) $\mu \leq \hat{\mu}\left(\theta^{l}\right)$ and $\gamma<1 /(1-\mu)$;

ii) $\mu \in\left(\hat{\mu}\left(\theta^{l}\right), \hat{\mu}\left(\theta^{h}\right)\right]$;

iii) $\mu>\hat{\mu}\left(\theta^{h}\right)$ and $\gamma<1 / \mu$.

Lemma 7 is the counterpart of Lemma 3 and shows this study's key insight that early information revelation is easier to achieve under delegation than under centralization. Recall that early information revelation under centralization could not be implemented, because of an irreconcilable tension between the rachet problem associated with the efficient agent and 
the take-the-money-and-run problem associated with the inefficient agent. Under delegation, the principal can, for two distinct reasons, deal with this tension more easily.

First, for $\mu \leq \hat{\mu}\left(\theta^{h}\right)$, the sequentially rational contract $\Phi_{2}^{D L}$ provides a rent to the agent regardless of his labor efficiency $\theta^{L}$. This second period rent acts as a "carrot" and alleviates the take-the-money-and-run problem, because the inefficient agent loses this future rent if he decides to run away in $t=2$. We note that, even though the rent in $t=2$ benefits both types, it is only the rent to the inefficient agent that alleviates dynamic incentives.

Second, for $\mu>\hat{\mu}\left(\theta^{l}\right)$, the sequentially rational contract $\Phi_{2}^{D L}$ reduces production when the capital input is reported to be inefficient. Hence, delegation enables the principal to commit credibly to a reduced production in $t=2$. This play-hardball effect differs from the carrot effect in that it reduces the ratchet problem rather than the take-the-money-and-run problem. Due to the credible production cut in $t=2$, the efficient agent's information rent for that period becomes smaller, which in turn makes the agent less reluctant to reveal his information in $t=1$.

Lemma 7 shows how these two strategic effects of delegation alleviate dynamic incentive problems. For $\mu<\hat{\mu}\left(\theta^{l}\right)$, delegation displays only the carrot effect that reduces the takethe-money-and-run problem. For $\mu>\hat{\mu}\left(\theta^{h}\right)$, delegation displays only the play-hardball effect that reduces the rachet problem. Notice that for these ranges of $\mu$, delegation can be implemented only if the capacity expansion parameter $\gamma$ is not too large. For a large $\gamma$, the carrot effect alone (when $\mu$ is small) cannot ease the tension between the two incentive problems - the rent in the second period is not large enough compared to the large transfer to the efficient agent. As a result, the inefficient agent cannot be discouraged from taking the large transfer in the first period by mimicking the efficient agent. Likewise, for a large $\gamma$, the play-hardball effect alone (when $\mu$ is large) cannot ease the tension between the two incentive problems - although the production cut for $t=2$ is credible, the probability that such a cut takes place is small as $\mu$ is large. A small chance of the production cut for $t=2$ leads to a large transfer to the efficient agent in $t=1$ for his truthful behavior. Then again, such a large transfer invites the inefficient agent's misrepresenting behavior.

For $\mu \in\left(\hat{\mu}\left(\theta^{l}\right), \hat{\mu}\left(\theta^{h}\right)\right)$, delegation is especially effective in inducing early information revelation. Within this range, delegation has both the carrot and play-hardball effect, and therefore targets both the ratchet and the take-the-money-and-run problem. As mentioned above, the rent in $t=2$ acting as a carrot discourages the inefficient agent to play the take-the-money-and-run strategy in $t=1$. In addition, due to the play-hardball effect, the 
principal can reduce the ratchet problem associated with the efficient agent, which allows the principal to lower the transfer in the first period to the efficient agent. The smaller transfer to the efficient agent, in turn, further discourages the inefficient from playing the take-themoney-and-run strategy. Notice that, within this range of $\mu$, delegation can implement separation in $t=1$ regardless of the capacity expansion parameter $\gamma$.

Although the previous lemma shows that early revelation is easier to implement under delegation, it does not say that it is actually optimal for the principal to do so. In order to address the optimality, we first derives the principal's maximum payoff under delegation when early information revelation is induced.

Proposition 2 Under delegation, the principal's maximum expected payoff with early information revelation is:

$$
\Pi^{D S}= \begin{cases}\mu \theta^{h}+\left(\widetilde{\theta}+\theta^{l}\right) \gamma & \text { if } \mu \leq \hat{\mu}\left(\theta^{l}\right) \text { and } \gamma<\frac{1}{1-\mu}, \\ \mu \theta^{h}+2 \mu\left(\theta^{h}+(1-\mu) \theta^{l}\right) \gamma & \text { if } \mu \in\left(\hat{\mu}\left(\theta^{l}\right), \hat{\mu}\left(\theta^{h}\right)\right], \\ \mu \theta^{h}+\mu\left(\theta^{h}+\theta^{l}\right) \gamma & \text { if } \mu>\hat{\mu}\left(\theta^{h}\right) \text { and } \gamma<\frac{1}{\mu} .\end{cases}
$$

The remaining task is to compare the payoffs $\Pi^{C P}$ in Proposition 1 with $\Pi^{D S}$ in Proposition 2. This comparison yields our main result:

Proposition 3 Let $\widetilde{\gamma} \equiv \frac{1}{1-\mu}$ and $\widehat{\gamma} \equiv \frac{\mu \theta^{h}-\theta^{l}}{\mu^{2} \Delta \theta}$. The optimal operational structure is as follows:

i) For $\mu \leq \hat{\mu}\left(\theta^{l}\right),\left\{\begin{array}{l}\text { delegation is optimal if } \gamma<\widetilde{\gamma} \text { and } \mu>\hat{\mu}(0), \\ \text { centralization is optimal otherwise. }\end{array}\right.$

ii) For $\mu \in\left(\hat{\mu}\left(\theta^{l}\right), \hat{\mu}\left(\theta^{h}\right)\right]$, delegation is optimal.

iv) If $\mu>\hat{\mu}\left(\theta^{h}\right),\left\{\begin{array}{l}\text { delegation is optimal if } \gamma<\hat{\gamma}, \\ \text { centralization is optimal otherwise. }\end{array}\right.$

Figure 1 below illustrates the result. Although centralization provides the principal with more control, delegation enables her to mitigate the dynamic incentive problems associated with limited commitment. The principal prefers delegation when the uncertainty about the operational efficiency is large. When, by contrast, such uncertainty is small, the principal prefers retaining direct control of the capital input to extract rents in the later period.

\section{[INSERT FIGURE 1 HERE]}


To summarize, the key trade-off in choosing the operational structure is between control vs. dynamic incentives. Centralization allows the principal more direct control, whereas delegation allows the principal to improve dynamic incentives and, thereby, induces the agent to reveal his private information earlier. As a result, delegation is optimal when information revelation is important relative to the loss of control.

\section{Extensions}

In this section, we extend our discussion in two directions. First, we examine the role of complementarity in production. We argue that the carrot effect is robust to the degree of complementarity, whereas the play-hardball effect reacts more sensitively to it. As a result we conclude that complementarity in production tends to favor delegation.

Second, we investigate how our results change when the organization under centralization has more commitment power than the organization under delegation in that, under centralization, the organization can offer a long-term contract but is susceptible to renegotiation. We show that centralization under large uncertainty remains suboptimal, despite its larger degree of commitment. We further argue how this extension enlarges the scope of our analysis and may be used to address questions about the optimal boundary of the firm by following the idea that decisions that are taken outside the firm tend to increase private information and limit the firm's commitment power.

\section{Role of Complementarity}

So far, we have assumed that the labor and the capital input are complements. In this subsection, we discuss the case in which the two inputs are substitutes. Consider the following fully substitutive production function:

$$
Q_{t}=q_{\lambda t}+q_{\kappa t}, \text { where } t=1,2 .
$$

In line with the previous section, the firm's production capacity is limited to $Q_{1}=2$ in the first period and expands to $Q_{2}=2 \gamma$ in the second period, where $\gamma>1$. That is, $q_{\lambda 1}+q_{\kappa 1} \in[0,2]$ in $t=1$ and $q_{\lambda 2}+q_{\kappa 2} \in[0,2 \gamma]$ in $t=2$.

We first show that, in our parsimonious symmetric setting, a fully substitutive production function allows the principal to circumvent the ratchet effect completely. As a result, centralization with the substitutive technology implements separation in $t=1$ at no cost, 
thus dominating delegation. ${ }^{15}$ Under centralization, the first period contract that induces early information revelation is: $\Phi_{1}^{C S}=\left\{q_{1}^{L}, T_{1}^{L}\right\}, L \in\{h, l\}$. The second period contract specifies the agent's labor input $q_{\lambda 2}^{L K}$ and the transfer $T_{2}^{L K}$, contingent on the revealed labor efficiency in $t=1$ and the true capital efficiency. Given $\Phi_{1}^{C S}$ in $t=1$, the following contract $\Phi_{2}^{C}$ in $t=2$ is sequential rational:

$$
\left(q_{\lambda 2}^{L K}, q_{\kappa 2}^{L K}\right)=\left\{\begin{array}{ll}
(2 \gamma, 0) & \text { if } \theta^{L}>\theta^{K} \\
(0,2 \gamma) & \text { otherwise. }
\end{array} ; \quad T_{2}^{L K}= \begin{cases}2\left(1-\theta^{L}\right) \gamma & \text { if } \theta^{L}>\theta^{K} \\
0 & \text { otherwise }\end{cases}\right.
$$

Because the agent's labor efficiency is fully revealed in $t=1$ and can be substituted by the capital input controlled by the principal, no labor input is used in $t=2$ unless the agent's labor input is more efficient than the capital input. With the outcome in (6), we examine whether there exists a contract $\Phi_{1}^{C S}$ that implements the agent's truthful report in $t=1$.

Proposition 4 Suppose the labor and the capital input are substitutes and symmetrical ex ante. Then, a separating contact $\Phi_{1}^{C S}$ exists and centralization is optimal.

With the substitutive technology, the principal can always replace the agent's labor input with the capital input. Therefore, under centralization, the agent must compete with the capital input controlled by the principal. In a symmetric setting, centralization allows the principal to replace the agent's expensive labor with the capital input whenever the agent misreports his efficiency. This allows the principal to circumvent the ratchet effect, because an efficient agent does not expect to obtain any rents from his labor input in $t=2$ if he reports to be inefficient. Hence, unlike in the case of complementary inputs, centralized operation is able to induce early information revelation at no cost.

We, however, emphasize that the result in Proposition 4 is due to the simplifying assumption that the input efficiencies are symmetrical ex ante. In order to discuss the robustness of the play-hardball and the carrot effect with regard to complementarity in production, we have to consider substitutive production in a slightly more general framework. In particular, we let the efficiencies be input specific. The agent's labor input efficiency in $t=1$ is $\theta_{\lambda}^{h}$ with probability $\mu$ and $\theta_{\lambda}^{l}$ with probability $1-\mu$, where $\Delta \theta_{\lambda}=\theta_{\lambda}^{h}-\theta_{\lambda}^{l}>0$. We keep the assumption that in $t=1$, the marginal cost of the capital input is commonly known to be

\footnotetext{
${ }^{15}$ As before, provided that no information revelation is induced in $t=1$, delegation cannot be optimal since it only provides an additional rent to the agent in $t=2$.
} 
1. In $t=2$, it is $\theta_{\kappa}^{h}$ with probability $\mu$ and $\theta_{\kappa}^{l}$ with probability $1-\mu$. Now if $\theta_{\lambda}^{l}>\theta_{\kappa}^{l}$ then the sequential rational contract (6) implies that $q_{\lambda}^{l l}$ is $2 \gamma$ rather than zero. As a result Proposition 4 no longer holds.

Under delegation, the first period separating contract, $\Phi_{1}^{D S}$, induces the principal to offer the second period contract, $\Phi_{2}^{D S}$, under the belief that $\theta_{\lambda}^{L}=\theta_{1}^{L}$, but not knowing the efficiency of the capital input $\theta_{\kappa}^{K}$. Hence, the sequentially rational contract, $\Phi_{2}^{D S}$, is a triplet $\left\{q_{\lambda 2}^{L K}, q_{\kappa 2}^{L K}, T_{2}^{L K}\right\}, K \in\{l, h\}$, contingent on the first period report $\theta_{1}^{L} \in\left\{\theta_{\lambda}^{h}, \theta_{\lambda}^{l}\right\}$. Given $\Phi_{1}^{D S}$ in $t=1, \Phi_{2}^{D S}$ in $t=2$ maximizes:

$$
\Pi_{2}^{D S}=\mu\left(q_{\lambda 2}^{L h}+q_{\kappa 2}^{L h}-T_{2}^{L h}\right)+(1-\mu)\left(q_{\lambda 2}^{L l}+q_{\kappa 2}^{L l}-T_{2}^{L l}\right),
$$

subject to the incentive constraint for the agent for revealing $\theta_{\kappa}^{K}$ :

$$
T_{2}^{L K}-\left(1-\theta_{\lambda}^{L}\right) q_{\lambda 2}^{L K}-\left(1-\theta_{\kappa}^{K}\right) q_{\kappa 2}^{L K} \geq T_{2}^{L K^{\prime}}-\left(1-\theta_{\lambda}^{L}\right) q_{\lambda 2}^{L K^{\prime}}-\left(1-\theta_{\kappa}^{h}\right) q_{\kappa 2}^{L K^{\prime}}, \forall K, K^{\prime} \in\{h, l\}
$$

and his individual rationality constraint:

$$
T_{2}^{L K}-\left(1-\theta_{\lambda}^{L}\right) q_{\lambda 2}^{L K}-\left(1-\theta_{\kappa}^{K}\right) q_{\kappa 2}^{L K} \geq 0, \forall L, K \in\{h, l\} .
$$

As usual, the incentive constraint of the agent when $\theta_{\kappa}^{K}=\theta_{\kappa}^{h}$ and the individual rationality constraint of the agent when $\theta_{\kappa}^{K}=\theta_{\kappa}^{l}$ are binding. Substitution of these constraints, yields that the sequentially rational inputs $q_{\lambda 2}^{L h}, q_{\kappa 2}^{L h}, q_{\lambda 2}^{L l}$, and $q_{\kappa 2}^{L l}$ maximize:

$$
\Pi_{2}^{D S}=\mu\left(\theta_{\lambda}^{L} q_{\lambda 2}^{L h}+\theta_{\kappa}^{h} q_{\kappa 2}^{L h}\right)+(1-\mu)\left[\theta_{\lambda}^{L} q_{\lambda 2}^{L l}+\left(\theta_{\kappa}^{l}-\frac{\mu}{1-\mu} \Delta \theta_{\kappa}\right) q_{\kappa 2}^{L l}\right],
$$

under the capacity restrictions: $q_{\lambda 2}^{L h}+q_{\kappa 2}^{L h} \leq 2 \gamma$ and $q_{\lambda 2}^{L l}+q_{\kappa 2}^{L l} \leq 2 \gamma$.

The second term in expression (7), representing the case when the capital input has the low efficiency $\theta_{\kappa}^{l}$, plays a crucial role for understanding the role of complementarity in establishing the play-hardball and the carrot effect. To explain, we separate out the second term in (7) as follows:

$$
\theta_{\lambda}^{L} q_{\lambda 2}^{L l}+\left(\theta_{\kappa}^{l}-\frac{\mu}{1-\mu} \Delta \theta_{\kappa}\right) q_{\kappa 2}^{L l}
$$

As can be seen from (8), when the capital efficiency is $\theta_{\kappa}^{l}$, the optimal usage of inputs is distorted towards labor, because the principal discounts the low efficiency $\theta_{\kappa}^{l}$ by the term $\mu \Delta \theta_{\kappa} /(1-\mu)$. This is because the agent's private information on the capital input reduces the principal's valuation of the capital input, which in turn results in the usual downward distortion of the capital input in production. 
With a complementary production technology, the reduced valuation of the capital input implies that the principal reduces both the capital input and the agent's labor input. This joint reduction constitutes the play-hardball effect that alleviates dynamic incentives. In a substitutive framework, the reduced efficiency of capital means, by contrast, that the principal substitutes capital in favor of labor. Thus, the reduction in the capital input leads to an increase rather than a reduction in the agent's labor input. Consequently, there is no play-hardball effect under a substitutive production technology.

Next, we argue that the carrot effect can survive under a substitutive technology. To see this, note that the binding incentive and individual rationality constraint imply that the input schedule, $q_{\lambda 2}^{L h}, q_{\kappa 2}^{L h}, q_{\lambda 2}^{L l}$, and $q_{\kappa 2}^{L l}$, yields the agent with labor efficiency $\theta_{\lambda}^{L}$ and capital efficiency $\theta_{\kappa}^{h}$ a rent of $\Delta \theta_{\kappa} q_{\kappa 2}^{L l}$. Now it follows directly from (8) that the principal's sequentially rational contract exhibits $q_{\kappa 2}^{l l}=2 \gamma$ if

$$
\theta_{\lambda}^{l}<\theta_{\kappa}^{l}-\frac{\mu}{1-\mu} \Delta \theta_{\kappa}
$$

In this case, the inefficient agent anticipates an expected rent of $2 \Delta \theta_{\kappa} \gamma$ in $t=2 .{ }^{16}$ This expected rent signifies exactly the carrot effect that discourages the inefficient agent from playing the take-the-money-and-run strategy.

Summarizing, when the inputs are substitutive, delegation can still exhibit a carrot effect, but the play-hardball effect is lost. As a result, the optimality of delegation becomes weaker. We conclude this subsection by stating this insight as follows.

Result Complementarity in operation shifts the trade-off between delegation and centralization in favor of delegation.

\section{Modes of Commitment: Organizational Boundary}

In our base model, the principal could only offer short-term contracts. This raises the question, whether such an extreme form of limited commitment is crucial for delegation to outperform centralization. In this subsection, we demonstrate that delegation is also optimal under less extreme forms of limited commitment and even holds when we relax the degree of limited commitment only for centralization. In particular, we study a centralized

\footnotetext{
${ }^{16}$ Note that if $\theta_{\lambda}^{l}=\theta_{\kappa}^{l}=\theta^{l}$ then the inequality never holds so that the carrot effect under these parameter constellations does not obtain. Extending our framework to incorporate type dependent efficiencies therefore allows us to show more clearly how and why the carrot effect obtains with a substitutive production function.
} 
structure in which the principal in the first period can commit to the contract for the second period, but if the second period contract turns out to be ex post inefficient, she renegotiates away the inefficiency. ${ }^{17}$ For the delegated structure, however, we continue to assume that the principal can only offer short-term contracts. In other words, we allow the organization under centralization to offer a long-term contract with a possibility of renegotiation, whereas under delegation the principal is still restricted to short-term contracting. Thus, centralization now has an additional advantage over delegation - it gives the principal more commitment.

We first derive the equilibrium outcome under long-term contracting with renegotiation. The renegotiation-proofness principle (e.g. Bolton and Dewatripont, 2005) ensures that there is no loss of generality in focusing on direct mechanisms that are renegotiation-proof. Our approach is, therefore, to derive first the optimal renegotiation-proof contracts under centralization for the two cases - pooling and separating in the first period. We next compare them to the principal's optimal payoff under delegation $\Pi^{D S}$ as derived in Proposition 2.

Under pooling, where no early information revelation is induced, the output levels in $t=1$ are $q_{1}^{h}=q_{1}^{l}=1$. At the end of the first period, the agent is still privately informed about his labor efficiency. Therefore, any renegotiation takes place with the principal's belief $\mu$ about the agent's labor efficiency. It is then straightforward to see that the principal's payoff is the same as the one in Proposition 1.

Hence, for pooling contracts the optimal short-term contract and the optimal renegotiationproof, long-term contract coincide. This, however, is not the case for separating contracts. Indeed, whereas first-period separation is not implementable with short-term contracts, it is implementable with a long-term contract under renegotiation. The reason is twofold. First, renegotiation-proof contracts enable the principal to pledge a strictly positive rent to the efficient agent. Second, they allow her to contractually prevent the inefficient agent from playing the take-the-money-and-run strategy.

In order to derive the optimal renegotiation-proof contract that induces first period separation, note first that for any such contract the output levels in $t=1$ are $q_{1}^{h}=1$ and $q_{1}^{l}=0$. Because these output levels reveal the agent's labor efficiency after $t=1$, the principal at the renegotiation stage is fully informed about the agent's labor efficiency and would renegotiate away any inefficiency in the second period production levels. Therefore and in line

\footnotetext{
${ }^{17}$ Contract theory views "renegotiation" as a rather mild form of limited commitment, because any sequentially rational contract is ex post efficient, whereas not every ex post efficient contract is sequentially rational.
} 
with short-term contracting, any renegotiation-proof contract with first period separation exhibits $q_{2}^{h h}=q_{2}^{h l}=q_{2}^{l l}=q_{2}^{l h}=\gamma$. The next lemma presents the principal's maximum payoff from a renegotiation-proof contract which induces first period separation by minimizing the wages that implements the aforementioned output schedule under the appropriate incentive constraints.

Lemma 8 Under centralization, the principal's optimal renegotiation-proof contract that induces early information revelation yields $\Pi_{r p}^{C S} \equiv \mu \theta^{h}+\left(\theta^{l}+\tilde{\theta}\right) \gamma$.

Although renegotiation-proof contracts under centralization enable the principal to induce the agent to reveal his labor efficiency in the first period, a direct comparison of the payoffs $\Pi^{D S}$ and $\Pi_{r p}^{C S}$ shows that, with large uncertainty, it is more costly to do so under centralization. The next proposition therefore demonstrates that, even if centralization involves more commitment, delegation still outperforms it.

Proposition 5 If the uncertainty for operational efficiency is large $\mu \in\left(\hat{\mu}\left(\theta^{l}\right), \hat{\mu}\left(\theta^{h}\right)\right]$, then delegation with the short-term contracts outperforms centralization with renegotiation-proof, long-term contracts.

According to the proposition above, our result that delegation outperforms centralization is robust to the ability of the organization to offer long-term contracts, unless centralization allows the use ex post inefficient contracts. As mentioned above, when the principal offers a long-term contract in the beginning of the first period, she no longer faces the takethe-money-and-run problem associated with the inefficient agent. Yet, the efficient agent's reluctance to reveal his information still has bite because he anticipates the principal's renegotiation. Thus, the play-hardball effect survives, and this explains why even with long term contracts centralization is suboptimal under large uncertainty. Because the previous subsection revealed that the play-hardball effect arises only under complementarity, the optimality of delegation now depends crucially on such complementarity. ${ }^{18}$

By following Williamson (1985)'s view that long-term contracts better characterize intrafirm transactions, whereas spot contracts better describe transactions in outsourcing relationships, Proposition 5 has relevance for the theory of the firm and organizational boundaries:

\footnotetext{
${ }^{18}$ Our result that complementarity favors delegation is in line with the property rights literature (e.g., Hart and Moore 1990) which argues that complementarity favors integration. To see this, note that, the agent under delegation controls both inputs, which Hart and Moore (1990) interpret as integration.
} 
Firms that operate under a large degree of uncertainty outsource more than firms that operate under more certainty. This insight may shed light on differences in operating structures of industry leaders and followers as alluded to in the introduction.

\section{Conclusion}

When organizations have limited commitment, dynamic incentive problems arise which limit the rate at which they can induce their agents to reveal private information. Our analysis shows that delegation mitigates these dynamic incentive problems and enables a more timely revelation of information. The drawback of delegation is however a loss of control.

These insights suggest a theory of optimal organizational design, which hinges on a tradeoff between dynamic incentives vs. control: Organizations benefit from delegation if timely information revelation is more valuable than the cost of losing control associated with the delegation. Our analysis shows that this is the case when uncertainty is large and matters. Moreover, delegation is especially helpful when there are complementarity in operations.

Taking a positive perspective, these insights yield a set of hypotheses to test our theory. Because the benefit of delegation stems from its ability to facilitate the revelation of persistent information, a first direct empirical prediction is that organizations with higher degree of delegation are more prevalent in environments where persistent private information matters and the organization's commitment abilities to protect its agents against a dynamic exploitation of such information is limited. Our analytical results yield the further empirical predictions that delegation is more prevalent in environments in which there is more uncertainty (in terms of our model, when $\mu$ is intermediate) and the differences in efficiency are potentially large (in terms of our model, when the efficiency gap $\Delta \theta$ is large). ${ }^{19}$ Moreover, our insight that complementarity in operations favors delegation provides testable implications about the nature of the tasks which organizations delegate. In particular, the delegation concerns especially those tasks which are most complementary with the tasks about which the agent has persistent private information from the outset. Moreover, these delegated tasks should give the agent access to additional private information. Especially this last prediction differentiates our theory of delegation from prevailing ones.

\footnotetext{
${ }^{19}$ To see this latter implication, note that defining $\Delta \hat{\mu} \equiv \hat{\mu}\left(\theta^{h}\right)-\hat{\mu}\left(\theta^{l}\right)$ and differentiating with respect to $\theta^{h}$ and $\theta^{l}$ yields, respectively, $\partial \Delta \hat{\mu} / \partial \theta^{h}=\theta^{l}\left(\theta^{h}-\theta^{l}\right)\left(3 \theta^{h}+\theta^{l}\right) /\left(2 \theta^{h^{2}}\left(\theta^{h}+\theta^{l}\right)^{2}\right)>0$ and $\partial \Delta \hat{\mu} / \partial \theta^{l}=$ $-\left(3 \theta^{h}+\theta^{l}\right)\left(\theta^{h}-\theta^{l}\right) /\left(2 \theta^{h}\left(\theta^{h}+\theta^{l}\right)^{2}\right)<0$. Moreover, the curve $\hat{\gamma}$ shifts up as well, when $\Delta \theta$ increases.
} 


\section{Appendix A: Proofs}

Proof of Lemma 1 For each $K \in\{h, l\}$, we solve problem $P^{K}$ :

$$
P^{K}: \max _{q_{2}^{h K}, q_{2}^{l K}, T_{2}^{h K}, T_{2}^{l K}} \mu\left(q_{2}^{h K}-T_{2}^{h K}+\theta^{K} q_{2}^{h K}\right)+(1-\mu)\left(q_{2}^{l K}-T_{2}^{l K}+\theta^{K} q_{2}^{l K}\right) \text {, s.t. (2) and (3). }
$$

As is standard, only the participation constraint in (2) with respect to the inefficient type $\theta^{l}$ and the incentive constraint in (3) with respect to the efficient type $\theta^{h}$ are binding. These constraints yield $T_{2}^{l K}=\left(1-\theta^{l}\right) q_{2}^{l K}$ and $T_{2}^{h K}=\left(1-\theta^{h}\right) q_{2}^{h K}+\Delta \theta q_{2}^{l K}$. After substituting these transfers into the objective function, we are left to solve:

$$
\max _{q_{2}^{h K}, q_{2}^{l K}} \mu\left(\theta^{h}+\theta^{K}\right) q_{2}^{h K}+(1-\mu)\left(\theta^{l}+\theta^{K}-[\mu /(1-\mu)] \Delta \theta\right) q_{2}^{l K}
$$

The objective function is increasing in $q_{2}^{h K}$. Hence, $q_{2}^{h K}=\gamma$. Likewise, the objective function is non-decreasing in $q_{2}^{l K}$ if and only if $\mu \leq \hat{\mu}\left(\theta^{K}\right)$. Thus, $q_{2}^{l K}=\gamma$ if $\mu \leq \hat{\mu}\left(\theta^{K}\right)$, and $q_{2}^{l K}=0$ otherwise. Because $\hat{\mu}\left(\theta^{l}\right)<\hat{\mu}\left(\theta^{h}\right)$ we have the three cases as specified in the lemma.

Proof of Proposition 1 For $\mu \leq \hat{\mu}\left(\theta^{l}\right)$, it follows from Lemma 1 that the sequentially rational contract yields the principal a second period payoff of $\Pi_{2}^{C P} \equiv \mu \Pi_{2}^{C P}\left(\theta^{h}\right)+(1-$ $\mu) \Pi_{2}^{C P}\left(\theta^{l}\right)=\left(\theta^{l}+\widetilde{\theta}\right) \gamma$. For $\mu \in\left(\hat{\mu}\left(\theta^{l}\right), \hat{\mu}\left(\theta^{h}\right)\right.$ this payoff is $\Pi_{2}^{C P} \equiv \mu(2-\mu)\left(\theta^{h}+\theta^{l}\right) \gamma$. For $\mu>\hat{\mu}\left(\theta^{h}\right)$, her payoff is $\Pi_{2}^{C P}=\mu\left(\theta^{h}+\widetilde{\theta}\right) \gamma$. These payoffs are independent of the first period contract $\Phi_{1}^{C P}$. The principal's optimal first period pooling contract $\Phi_{1}^{C P}$, therefore, simply maximizes $\Pi_{1}^{C P} \equiv q_{1}-T_{1}$ subject to the participation constraints of the agent over both periods. Lemma 1 shows that the inefficient agent does not get a rent from the sequentially rational contract. His participation constraint is, therefore, $T_{1}-\left(1-\theta^{l}\right) q_{1} \leq 0$. Given this constraint, the contract $q_{1}=1$ and $T_{1}=1-\theta^{l}$ maximizes $\Pi_{1}^{C P}$. Because this contract automatically satisfies the participation of the efficient agent, it is optimal. It yields the principal the payoff $\Pi_{1}^{C P}=\theta^{l}$ in $t=1$. A simple summation of $\Pi_{1}^{C P}+\Pi_{2}^{C P}$ yields the expression for $\Pi^{C P}$.

Proof of Lemma 2 Given the agent's report on $\theta^{L}$ in $t=1$, the principal believes to face the agent of type $\theta^{L} \in\left\{\theta^{h}, \theta^{l}\right\}$ with certainty. Thus, she believes that the agent's participation constraint is $T_{2}^{L K}-\left(1-\theta^{L}\right) q_{2}^{L K} \geq 0$. Maximizing the principal's profits $2 q_{2}^{L K}-$ $T_{2}^{L K}-\left(1-\theta^{K}\right) q_{2}^{L K}$ subject to the agent's participation constraint yields the result. 
Proof of Lemma 3 Given that the agent anticipates the second period contract $\Phi_{2}^{C S}$, the contract $\Phi_{1}^{C S}$ is incentive compatible if

$$
\begin{aligned}
& U_{1}^{C}\left(q_{1}^{h}, T_{1}^{h} \mid \theta^{h}\right)+\mu U_{2}^{C}\left(q_{2}^{h h}, T_{2}^{h h} \mid \theta^{h}\right)+(1-\mu) U_{2}^{C}\left(q_{2}^{h l}, T_{2}^{h l} \mid \theta^{h}\right) \\
\geq & U_{1}^{C}\left(q_{1}^{l}, T_{1}^{l} \mid \theta^{h}\right)+\mu \max \left\{0, U_{2}^{C}\left(q_{2}^{l h}, T_{2}^{l h} \mid \theta^{h}\right)\right\}+(1-\mu) \max \left\{0, U_{2}^{C}\left(q_{2}^{l l}, T_{2}^{l l} \mid \theta^{h}\right)\right\} .
\end{aligned}
$$

and

$$
\begin{aligned}
& U_{1}^{C}\left(q_{1}^{l}, T_{1}^{l} \mid \theta^{l}\right)+\mu U_{2}^{C}\left(q_{2}^{l h}, T_{2}^{l h} \mid \theta^{l}\right)+(1-\mu) U_{2}^{C}\left(q_{2}^{l l}, T_{2}^{l l} \mid \theta^{l}\right) \\
\geq & U_{1}^{C}\left(q_{1}^{h}, T_{1}^{h} \mid \theta^{l}\right)+\mu \max \left\{0, U_{2}^{C}\left(q_{2}^{h h}, T_{2}^{h h} \mid \theta^{l}\right)\right\}+(1-\mu) \max \left\{0, U_{2}^{C}\left(q_{2}^{h l}, T_{2}^{h l} \mid \theta^{l}\right)\right\},
\end{aligned}
$$

where the "max" expression captures the agent's possibility to play his "take-the-moneyand-run" strategy and reject the second period contract. Using $q_{1}^{h}=1, q_{1}^{l}=0$, and Lemma 2 to substitute out the equilibrium quantities, the incentive constraints simplify to:

$$
\begin{gathered}
T_{1}^{h}-\left(1-\theta^{h}\right) \geq T_{1}^{l}+\gamma \Delta \theta, \\
T_{1}^{l} \geq T_{1}^{h}-\left(1-\theta^{l}\right) .
\end{gathered}
$$

These constraints imply that:

$$
\left(1-\theta^{h}\right)+\gamma \Delta \theta \leq T_{1}^{h}-T_{1}^{l} \leq 1-\theta^{l}
$$

But, because $\gamma>1$ implies $1-\theta^{h}+\gamma \Delta \theta>1-\theta^{h}+\Delta \theta=1-\theta^{l}$, the former string of inequalities cannot hold. Hence, no values for $T_{1}^{h}$ and $T_{1}^{l}$ exist that satisfy both (A1) and (A2).

Proof of Lemma 4 In $t=2$, the sequentially rational contract $\Phi_{2}^{D P}$ describes pairs $\left\{\left(q_{2}^{j}, T_{2}^{j}\right)\right\}^{j \in\{h, m, l\}}$ and maximizes

$$
\sum_{j} \varphi^{j}\left(2 q_{2}^{j}-T^{j}\right) \text { s.t. } T^{j}-\left(2-\Theta^{j}\right) q_{2}^{j} \geq 0, T^{j}-\left(2-\Theta^{j}\right) q_{2}^{j} \geq T^{j^{\prime}}-\left(2-\Theta^{j}\right) q_{2}^{j^{\prime}}, \forall j, j^{\prime} .
$$

As usual, the participation constraint for the most inefficient type $\Theta^{l}$, and the incentive constraints for the more efficient adjacent types, $\Theta^{h}$ (for $\Theta^{m}$ ) and $\Theta^{m}$ (for $\Theta^{l}$ ), are binding provided that this solution yields a non-decreasing schedule $q_{2}^{h} \geq q_{2}^{m} \geq q_{2}^{l}$.

The binding constraints imply, successively, $T^{l}=\left(2-\Theta^{l}\right) q_{2}^{l}, T^{m}=\left(2-\Theta^{m}\right) q_{2}^{m}+\left(\Theta^{m}-\right.$ $\left.\Theta^{l}\right) q_{2}^{l}$, and $T^{h}=\left(2-\Theta^{h}\right) q_{2}^{h}+\left(\Theta^{h}-\Theta^{m}\right) q_{2}^{m}+\left(\Theta^{m}-\Theta^{l}\right) q_{2}^{l}$. Substitution into the objective function and using the definition of $\Theta^{j}$ and $\phi^{j}$ implies that we maximize:

$$
2 \mu^{2} \theta^{h} q_{2}^{h}+\left[2(1-\mu) \mu\left(\theta^{h}+\theta^{l}\right)-\mu^{2} \Delta \theta\right] q_{2}^{m}+\left[2(1-\mu)^{2} \theta^{l}-2 \mu(1-\mu) \Delta \theta-\mu^{2} \Delta \theta\right] q_{2}^{l} .
$$


Maximizing the expression with respect to $q_{2}^{h} \in\{0, \gamma\}$ yields $q_{2}^{h}=\gamma$. Maximizing the expression with respect to $q_{2}^{m} \in\{0, \gamma\}$, we get $q_{2}^{m}=\gamma$ if $2 \mu(1-\mu)\left(\theta^{h}+\theta^{l}\right) \geq \mu^{2} \Delta \theta$, which is equivalent to $\mu \leq \bar{\mu}$, and $q_{2}^{m}=0$ otherwise with $\bar{\mu} \equiv\left[2\left(\theta^{h}+\theta^{l}\right)\right] /\left[3 \theta^{h}+\theta^{l}\right]$. Finally, maximizing the expression with respect to $q_{2}^{l} \in\{0, \gamma\}$, we get $q_{2}^{l}=\gamma$ if $(1-\mu)^{2} \theta^{l} \geq 2 \mu(1-\mu) \Delta \theta / 2+$ $\mu^{2} \Delta \theta / 2$, which is equivalent to $\mu \leq \underline{\mu}$, and $q_{2}^{l}=0$ otherwise with $\underline{\mu} \equiv 1-\sqrt{\Delta \theta /\left[\theta^{h}+\theta^{l}\right]}$.

The schedule $q_{2}^{h}, q_{2}^{m}, q_{2}^{l}$ is monotone if $\bar{\mu} \geq \underline{\mu}$, because it then follows that $q_{2}^{l}=\gamma$ implies $q_{2}^{m}=\gamma$. To see that $\bar{\mu} \geq \underline{\mu}$, note that it is equivalent to $2\left(\theta^{h}+\theta^{l}\right) /\left(3 \theta^{h}+\theta^{l}\right) \geq$ $1-\sqrt{\Delta \theta /\left(\theta^{h}+\theta^{l}\right)}$, which is equivalent to $\sqrt{\Delta \theta /\left(\theta^{h}+\theta^{l}\right)} \geq \Delta \theta /\left(3 \theta^{h}+\theta^{l}\right)$. But the last inequality holds due to $\sqrt{\Delta \theta /\left(\theta^{h}+\theta^{l}\right)}>\Delta \theta /\left(\theta^{h}+\theta^{l}\right)>\Delta \theta /\left(3 \theta^{h}+\theta^{l}\right)$.

For $\mu \leq \underline{\mu}$, the sequentially rational contract yields the principal a second period payoff of $\Pi_{2}=2 \theta^{l} \gamma$ and the inefficient agent $\theta^{L}=\theta^{l}$ expects a payoff $\mu \Delta \theta \gamma$, because he becomes type $\Theta^{m}$ with probability $\mu$. For $\mu \in(\underline{\mu}, \bar{\mu}]$ the principal's payoff is $\Pi_{2}=\left[1-(1-\mu)^{2}\right]\left(\theta^{h}+\theta^{l}\right) \gamma$ and the inefficient agent expects a zero payoff. For $\mu>\bar{\mu}$, the principal's payoff is $\Pi_{2}=$ $2 \mu^{2} \theta^{h} \gamma$ and the inefficient agent expects a zero payoff. These payoffs are independent of the first period contract $\Phi_{1}^{D P}$. The principal's optimal first period pooling contract $\Phi_{1}^{D P}$, therefore, maximizes $\Pi_{1}^{D P} \equiv q_{1}-T_{1}$ subject to the agent's participation constraints over both periods. The inefficient agent receives a rent in $t=2$ only for $\mu \leq \mu$. In this case, his participation constraint is $T_{1}-\left(1-\theta^{l}\right) q_{1}+\mu \Delta \theta \gamma \leq 0$ so that the optimal first period contract is $q_{1}=1$ and $T_{1}=\left(1-\theta^{l}\right)-\mu \Delta \theta \gamma$. It yields the principal the payoff $\theta^{l}+\mu \Delta \theta \gamma$ in $t=1$. For $\mu>\mu$, the inefficient agent does not expect a rent from the sequentially rational contract so that his participation constraint is $T_{1}-\left(1-\theta^{l}\right) q_{1} \leq 0$. Hence, the optimal first period contract is $q_{1}=1$ and $T_{1}=1-\theta^{l}$. It yields the principal the payoff $\theta^{l}$ in $t=1$. Adding the payoffs over both period yields the principal's maximum payoff from a pooling contract under delegation as $\Pi^{D P}=\theta^{l}+2 \theta^{l} \gamma$ if $\mu \leq \underline{\mu}, \Pi^{D P}=\theta^{l}+\left[1-(1-\mu)^{2}\right]\left(\theta^{h}+\theta^{l}\right) \gamma$ if $\mu \in(\mu, \bar{\mu}]$, and $\Pi^{D P}=\theta^{l}+2 \mu^{2} \theta^{h} \gamma$ otherwise.

Next, we show that $\Delta \Pi^{P} \equiv \Pi^{C P}-\Pi^{D P} \geq 0$ for all $\mu \in[0,1]$. In order to compute $\Delta \Pi^{P}$, note that $\underline{\mu}<\hat{\mu}\left(\theta^{l}\right)$, which follows from:

$$
\hat{\mu}\left(\theta^{l}\right)-\underline{\mu}=\frac{2 \theta^{l}}{\theta^{h}+\theta^{l}}-1+\sqrt{\frac{\Delta \theta}{\theta^{h}+\theta^{l}}}=-\frac{\Delta \theta}{\theta^{h}+\theta^{l}}+\sqrt{\frac{\Delta \theta}{\theta^{h}+\theta^{l}}},
$$

which is positive because the fraction is smaller than 1 . Moreover, $\bar{\mu}>\hat{\mu}\left(\theta^{h}\right)$, which follows from:

$$
\bar{\mu}-\hat{\mu}\left(\theta^{h}\right)=\frac{2\left(\theta^{h}+\theta^{l}\right)}{3 \theta^{h}+\theta^{l}}-\frac{\theta^{l}+\theta^{h}}{2 \theta^{h}}=\frac{\theta^{h^{2}}-\theta^{l^{2}}}{2 \theta^{h}\left(3 \theta^{h}+\theta^{l}\right)}>0 .
$$


Using Proposition 1, we can subsequently derive $\Delta \Pi^{P}$ as:

$$
\Delta \Pi^{P}= \begin{cases}0 & \text { if } \mu \leq \mu \\ (1-\mu)\left(2 \theta^{l}-\mu\left(\theta^{h}+\theta^{l}\right)\right) \gamma & \text { if } \mu<\mu \leq \hat{\mu}\left(\theta^{l}\right) \\ 0 & \text { if } \hat{\mu}\left(\theta^{l}\right)<\mu \leq \hat{\mu}\left(\theta^{h}\right) \\ \mu\left(2 \mu \theta^{h}-\left(\theta^{h}-\theta^{l}\right)\right) \gamma & \text { if } \hat{\mu}\left(\theta^{h}\right)<\mu \leq \bar{\mu} \\ (1-\mu) \mu\left(\theta^{h}+\theta^{l}\right) \gamma & \text { if } \mu>\bar{\mu} .\end{cases}
$$

These terms are all non-negative, because $\mu \leq \hat{\mu}\left(\theta^{l}\right)$ implies $2 \theta^{l} \geq \mu\left(\theta^{h}+\theta^{l}\right)$ and $\mu>\hat{\mu}\left(\theta^{h}\right)$ implies $2 \mu \theta^{h}>\theta^{h}-\theta^{l}$.

Proof of Lemma 5 Given the report on $\theta^{L}$ in $t=1$, the principal's problem is equivalent to a static contracting problem where there is private information about $\theta^{K} \in\left\{\theta^{h}, \theta^{l}\right\}$. As in Lemma 1, the participation of the agent when $\theta^{K}=\theta^{l}$ and the incentive compatibility constraint when $\theta^{K}=\theta^{h}$ are binding. This yields transfers:

$$
T_{2}^{L l}=\left(2-\theta^{L}-\theta^{l}\right) q_{2}^{L l} \text { and } T_{2}^{L h}=\left(2-\theta^{L}-\theta^{h}\right) q_{2}^{L h}+\Delta \theta q_{2}^{L l} .
$$

Substituting these variables in the principal's objective function yields:

$$
\begin{aligned}
& \mu\left[2 q_{2}^{L h}-\left(2-\theta^{L}-\theta^{h}\right) q_{2}^{L h}-\Delta \theta q_{2}^{L l}\right]+(1-\mu)\left[2 q_{2}^{L l}-\left(2-\theta^{L}-\theta^{l}\right) q_{2}^{L l}\right] \\
= & \mu\left[\theta^{L}+\theta^{h}\right] q_{2}^{L h}+\left[(1-\mu)\left(\theta^{L}+\theta^{l}\right)-\mu \Delta \theta\right] q_{2}^{L l} .
\end{aligned}
$$

Maximizing this expression for $q_{2}^{L h} \in\{0, \gamma\}$ yields $q_{2}^{L h}=\gamma$. Maximizing the expression for $q_{2}^{L l} \in\{0, \gamma\}$ yields $q_{2}^{L l}=\gamma$ if $(1-\mu)\left(\theta^{a}+\theta^{l}\right) \geq \mu \Delta \theta$, which is equivalent to $\mu \leq \hat{\mu}\left(\theta^{a}\right)$, and $q_{2}^{L l}=0$ otherwise. Because $\hat{\mu}\left(\theta^{h}\right)>\hat{\mu}\left(\theta^{l}\right)$, the lemma follows.

Proof of Lemma 6 After misreporting $\theta^{L}=\theta^{l}$ as $\theta^{h}$ in $t=1$, the principal offers the $\theta^{l}$ agent the sequential rational contract $\Phi_{2}^{D S}$, as specified in Lemma 5 . When accepting it, the $\theta^{l}$ agent receives the payoff $T_{2}^{h K^{\prime}}-\left(1-\theta^{l}\right) q_{2}^{h K^{\prime}}-\left(1-\theta^{K}\right) q_{2}^{h K^{\prime}}$ when his true capital costs are $\theta^{K} \in\{h, l\}$ and he reports them as $\theta^{K^{\prime}}$ in $t=2$. It is straightforward to check that, for the sequential rational contract $\Phi_{2}^{D S}$ these payoffs are all non-positive for all combinations $\left(K, K^{\prime}\right) \in\{h, l\}^{2}$ and any $\mu \in[0,1]$ so that it is optimal for the $\theta^{l}$ agent to reject the contract $\Phi_{2}^{D S}$ in $t=2$.

After misreporting $\theta^{L}=\theta^{h}$ as $\theta^{l}$ in $t=1$, the $\theta^{h}$ agent receives the contract $\Phi_{2}^{D S}$ as specified in Lemma 5. That is, he receives the payoff $T_{2}^{l K^{\prime}}-\left(1-\theta^{h}\right) q_{2}^{l K^{\prime}}-\left(1-\theta^{K}\right) q_{2}^{l K^{\prime}}$ from 
accepting the contract when his capital cost is $\theta^{K}$ and he reports them as $\theta^{K^{\prime}}$ after accepting the contract $\Phi_{2}^{D S}$. It is straightforward to check that, for the contract $\Phi_{2}^{D S}$, the $\theta^{h}$ agent always has a weakly higher payoff from reporting truthfully that $\theta^{K^{\prime}}=\theta^{K}$ and this payoff is non-negative.

Proof of Lemma 7 Following Lemma 5 we have to distinguish three different cases:

Case $i) \mu \leq \hat{\mu}\left(\theta^{l}\right)$ : From Lemma 5 it follows that the agent with the high labor efficiency $\theta^{h}$ receives an expected rent $\mu \Delta \theta \gamma$ if he truthfully reports $\theta_{1}^{L}=\theta^{h}$ in $t=1$. If he misreports by $\theta_{1}^{L}=\theta^{l}$ then, by Lemmas 5 and 6 , the agent receives a rent in $t=2$ of $2 \Delta \theta \gamma$ if $\theta^{K}=\theta^{h}$, and $\Delta \theta \gamma$ if $\theta^{K}=\theta^{l}$. Thus, $\Phi_{1}^{D S}$ is incentive compatible to the agent with labor efficiency $\theta^{h}$ if:

$$
T_{1}^{h}-\left(1-\theta^{h}\right)+\mu \Delta \theta \gamma \geq T_{1}^{l}+\mu 2 \Delta \theta \gamma+(1-\mu) \Delta \theta \gamma
$$

Likewise, it follows from Lemmas 5 and 6 that, regardless of his report in $t=1$, the agent with the low labor efficiency $\theta^{l}$ receives no rent in $t=2$. Thus, $\Phi_{1}^{D S}$ is incentive compatible to the agent with labor efficiency $\theta^{l}$ if:

$$
T_{1}^{l}+\mu \Delta \theta \gamma \geq T_{1}^{h}-\left(1-\theta^{l}\right)
$$

Combining the two conditions above shows that $\Phi_{1}^{D S}$ is incentive compatible when:

$$
\left(1-\theta^{h}\right)+\Delta \theta \gamma \leq T_{1}^{h}-T_{1}^{l} \leq\left(1-\theta^{l}\right)+\mu \Delta \theta \gamma
$$

Implementability requires $1-\theta^{h}+\Delta \theta \gamma<1-\theta^{l}+\mu \Delta \theta \gamma$ which holds when $\gamma<1 /(1-\mu)$.

Case $i i) \mu \in\left(\hat{\mu}\left(\theta^{l}\right), \hat{\mu}\left(\theta^{h}\right)\right]$ : From Lemma 5 and Lemma 6 it follows that the agent with labor efficiency $\theta^{h}$ receives in $t=2$ an expected information rent of $\mu \Delta \theta \gamma$ irrespective of his report in $t=1$. Thus, $\Phi_{1}^{D S}$ is incentive compatible to the agent with labor efficiency $\theta^{h}$ if

$$
T_{1}^{h}-\left(1-\theta^{h}\right)+\mu \Delta \theta \gamma \geq T_{1}^{l}+\mu \Delta \theta \gamma
$$

Likewise, it follows from Lemma 5 that the agent with low labor efficiency $\theta^{l}$ receives an expected rent $\mu \Delta \theta \gamma$ in $t=2$ from reporting $\theta^{l}$. If he reports $\theta^{h}$ instead then, by Lemma 6 , he receives no rent in $t=2$. Thus, $\Phi_{1}^{D S}$ is incentive compatible to the agent with labor efficiency $\theta^{l}$ if:

$$
T_{1}^{l}+\mu \Delta \theta \gamma \geq T_{1}^{h}-\left(1-\theta^{l}\right)
$$


Combining the previous two conditions shows that $\Phi_{1}^{D S}$ is incentive compatible exactly when:

$$
1-\theta^{h} \leq T_{1}^{h}-T_{1}^{l} \leq 1-\theta^{l}+\mu \Delta \theta \gamma .
$$

Hence, implementability requires $1-\theta^{h}<1-\theta^{l}+\mu \Delta \theta \gamma$ which is always the case.

Case iii) $\mu>\hat{\mu}\left(\theta^{h}\right)$ : From Lemma 5 it follows that the agent with labor efficiency $\theta^{h}$ receives no rent in $t=2$ if he truthfully reports $\theta_{1}^{L}=\theta^{h}$ in $t=1$. If, he misreports $\theta^{L}$ as $\theta^{l}$ then Lemma 6 and 5 imply that he receives a rent in $t=2$ of $\Delta \theta \gamma$ in $t=2$ if $\theta^{K}=\theta^{h}$, and no rent if $\theta^{K}=\theta^{l}$. Thus, $\Phi_{1}^{D S}$ is incentive compatible to the agent with labor efficiency $\theta^{h}$ if

$$
T_{1}^{h}-\left(1-\theta^{h}\right) \geq T_{1}^{l}+\mu \Delta \theta \gamma .
$$

Likewise, it follows from Lemma 5 that, irrespective of his report in $t=2$, the agent with labor efficiency $\theta^{l}$ receives no rent in $t=2$. Thus, $\Phi_{1}^{D S}$ is incentive compatible to the agent with labor efficiency $\theta^{l}$ if

$$
T_{1}^{l} \geq T_{1}^{h}-\left(1-\theta^{l}\right)
$$

Combining the previous two conditions shows that $\Phi_{1}^{D S}$ is incentive compatible exactly when:

$$
\left(1-\theta^{h}\right)+\mu \Delta \theta \gamma \leq T_{1}^{h}-T_{1}^{l} \leq\left(1-\theta^{l}\right) .
$$

Implementability requires $1-\theta^{h}+\mu \Delta \theta \gamma<1-\theta^{l}$ which holds when $\gamma<1 / \mu$.

Proof of Proposition 2 A first period contract with early information revelation $\Phi_{1}^{D S}$ exhibits $q_{1}^{h}=1$ and $q_{1}^{l}=0$ and leads to the sequentially rational contract $\Phi_{2}^{D S}$ as specified in Lemma 5. The principal's only remaining degrees of freedom are the first period transfers $T_{1}^{h}$ and $T_{1}^{l}$. Optimal transfers $T_{1}^{h}$ and $T_{1}^{l}$ minimize the principal's expected transfer:

$$
E\left[T_{1}\right] \equiv \mu T_{1}^{h}+(1-\mu) T_{1}^{l},
$$

and we are left to determine $T_{1}^{h}$ and $T_{1}^{l}$. For $\mu \leq \hat{\mu}\left(\theta^{l}\right)$, the principal minimizes $E\left[T_{1}\right]$ under the incentive constraints (A3) and the participation constraints,

$$
T_{1}^{h}-\left(1-\theta^{h}\right)+\mu \Delta \theta \gamma \geq 0 \quad \text { and } \quad T_{1}^{l}+\mu \Delta \theta \gamma \geq 0 .
$$

At the optimum the participation constraint of type $\theta^{l}$ and the incentive constraint of type $\theta^{h}$ are binding. This yields $T_{1}^{h}=1-\theta^{h}+(1-\mu) \Delta \theta \gamma$ and $T_{1}^{l}=-\mu \Delta \theta \gamma$. The principal's payoff is $\Pi^{D S}=\mu \theta^{h}+\left(\tilde{\theta}+\theta^{l}\right) \gamma$. 
For $\hat{\mu}\left(\theta^{l}\right) \leq \mu \leq \hat{\mu}\left(\theta^{h}\right)$, the principal minimizes $E\left[T_{1}\right]$ under the incentive constraints (A4) and the participation constraints,

$$
T_{1}^{h}-\left(1-\theta^{h}\right)+\mu \Delta \theta \gamma \geq 0 \quad \text { and } \quad T_{1}^{l} \geq 0 .
$$

At the optimum the participation constraint of type $\theta^{l}$ and $\theta^{h}$ are binding. This yields $T_{1}^{h}=1-\theta^{h}-\mu \Delta \theta \gamma$ and $T_{1}^{l}=0$. The principal's payoff is $\Pi^{D S}=\mu\left(\theta^{h}+\mu \Delta \theta \gamma\right)+(1-(1-$ $\left.\mu)^{2}\right)\left(\theta^{h}+\theta^{l}\right) \gamma=\mu \theta^{h}+2 \mu\left(\theta^{h}+(1-\mu) \theta^{l}\right) \gamma$.

For $\mu>\hat{\mu}\left(\theta^{h}\right)$ we minimize $E T_{1}$ under the incentive constraints (A5) and the participation constraints,

$$
T_{1}^{h}-\left(1-\theta^{h}\right) \geq 0 \quad \text { and } \quad T_{1}^{l} \geq 0 .
$$

At the optimum the participation constraint of type $\theta^{l}$ and the incentive constraint of type $\theta^{h}$ are binding. This yields $T_{1}^{h}=1-\theta^{h}+\mu \Delta \theta \gamma$ and $T_{1}^{l}=0$. The principal's payoff is $\Pi^{D S}=\mu\left(\theta^{h}-\mu \Delta \theta \gamma\right)+\left[2 \mu^{2} \theta^{h}+(1-\mu) \mu\left(\theta^{h}+\theta^{l}\right) \gamma=\mu \theta^{h}+\mu\left(\theta^{h}+\theta^{l}\right) \gamma\right.$.

Proof of Proposition 3 By Lemma 4, centralization is optimal whenever first period separation under delegation is not implementable. We therefore only need to compare $\Pi^{C P}$ with $\Pi^{D S}$. From Proposition 1 and 2 , we compute $\Delta \Pi=\Pi^{C P}-\Pi^{D S}$ whenever first period separation under delegation is implementable. For $\mu \leq \hat{\mu}\left(\theta^{l}\right)$ and $\gamma<1 /(1-\mu)$ it follows $\Delta \Pi=\theta^{l}-\mu \theta^{h}$, which is positive exactly when $\mu<\theta^{l} / \theta^{h}=\hat{\mu}(0)$. Hence, if $\mu<\hat{\mu}(0)$ centralization is optimal irrespective of $\gamma$. Instead, for $\hat{\mu}(0)<\mu \leq \hat{\mu}\left(\theta^{l}\right)$, delegation is optimal whenever it is implementable which is the case for $\gamma \geq \widetilde{\gamma} \equiv 1 /(1-\mu)$. For $\hat{\mu}\left(\theta^{l}\right)<\mu \leq \hat{\mu}\left(\theta^{h}\right)$, we have $\Delta \Pi=\theta^{l}-\mu \theta^{h}-\mu^{2} \Delta \theta<-\mu^{2} \Delta \theta<0$, where the first inequality follows because $\mu \geq$ $\hat{\mu}\left(\theta^{l}\right)>\hat{\mu}(0)$. Hence, delegation is optimal whenever implementable and implementability is always ensured. For $\mu>\hat{\mu}\left(\theta^{h}\right)$ and $\gamma<1 / \mu$ we have $\Delta \Pi=\theta^{l}-\mu \theta^{h}+\mu\left(\tilde{\theta}-\theta^{l}\right) \gamma=$ $\theta^{l}-\mu \theta^{h}+\mu^{2} \Delta \theta \gamma$. This is negative exactly when $\gamma<\widehat{\gamma} \equiv\left(\mu \theta^{h}-\theta^{l}\right) /\left(\mu^{2} \Delta \theta\right)$. Because $\left(\mu \theta^{h}-\theta^{l}\right) /\left(\mu^{2} \Delta \theta\right)<\mu\left(\theta^{h}-\mu \theta^{l}\right) /\left(\mu^{2} \Delta \theta\right)<1 / \mu, \Delta \Pi<0$ implies $\gamma<1 / \mu$ so that first period separation is implementable.

Proof of Proposition 4 From the expressions in (6), the agent's second period rent is zero in any case. Thus, $\Phi_{1}^{C S}$ is incentive compatible if:

$$
T_{1}^{h}-\left(1-\theta^{h}\right) \geq T_{1}^{l} \quad \text { and } \quad T_{1}^{l} \geq T_{1}^{h}-\left(1-\theta^{l}\right) .
$$


Combining the previous two conditions shows that $\Phi_{1}^{C S}$ is incentive compatible exactly when:

$$
1-\theta^{h} \leq T_{1}^{h}-T_{1}^{l} \leq 1-\theta^{l},
$$

implying that implementability requires $1-\theta^{h}<1-\theta^{l}$ which is always the case.

In $t=1$, the principal minimizes the expected transfer:

$$
E\left[T_{1}^{L}\right]=\mu T_{1}^{h}+(1-\mu) T_{1}^{l},
$$

subject to the efficient agent's truth-telling incentive and the inefficient agent's participation:

$$
T_{1}^{h}-\left(1-\theta^{h}\right) \geq T_{1}^{l} \quad \text { and } \quad T_{1}^{l} \geq 0 .
$$

Again, these constraints are binding in the optimal contract, and $T_{1}^{h}=1-\theta^{h}$ and $T_{1}^{l}=0$. It follows that $\Psi=C$ is optimal.

Proof of Lemma 8 With separating in the first period, the principal learns the agent type after the first period so that any renegotiation-proof contract is "first-best efficient" in the second period and exhibits: $q_{2}^{h h}=q_{2}^{h l}=q_{2}^{l l}=q_{2}^{l h}=\gamma$. We compute the minimum expected wage transfer for implementing the output schedule: $q_{1}^{h}=1$ and $q_{1}^{l}=0$ in $t=1$, and $q_{2}^{h h}={ }_{2} q^{h l}=q_{2}^{l l}=q_{2}^{l h}=\gamma$ in $t=2$. The expected wage transfer is:

$$
\mu W_{e}^{h}+(1-\mu) W_{e}^{l}
$$

where $W_{e}^{L} \equiv T_{1}^{L}+\mu T_{2}^{L h}+(1-\mu) T_{2}^{L l}$ represents the expected wage transfer conditional on the agent's labor efficiency $\theta^{L}$ with $L \in\{h, l\}$.

In order to induce first period separation, the principal has to respect the following incentive compatibility conditions:

$$
\begin{aligned}
& T_{1}^{h}-\left(1-\theta^{h}\right) q_{1}^{h}+\mu\left(T_{2}^{h h}-\left(1-\theta^{h}\right) q_{2}^{h h}\right)+(1-\mu)\left(T_{2}^{h l}-\left(1-\theta^{h}\right) q_{2}^{h l}\right) \\
\geq & T_{1}^{l}-\left(1-\theta^{h}\right) q_{1}^{l}+\mu\left(T_{2}^{l h}-\left(1-\theta^{h}\right) q_{2}^{l h}\right)+(1-\mu)\left(T_{2}^{l l}-\left(1-\theta^{h}\right) q_{2}^{l l}\right),
\end{aligned}
$$

for the agent whose labor efficiency is $\theta^{h}$, and

$$
\begin{aligned}
& T_{1}^{l}-\left(1-\theta^{l}\right) q_{1}^{l}+\mu\left(T_{2}^{l h}-\left(1-\theta^{l}\right) q_{2}^{l h}\right)+(1-\mu)\left(T_{2}^{l l}-\left(1-\theta^{l}\right) q_{2}^{l l}\right) \\
\geq & T_{1}^{h}-\left(1-\theta^{l}\right) q_{1}^{h}+\mu\left(T_{2}^{h h}-\left(1-\theta^{l}\right) q_{2}^{h h}\right)+(1-\mu)\left(T_{2}^{h l}-\left(1-\theta^{l}\right) q_{2}^{h l}\right),
\end{aligned}
$$


for the agent whose labor efficiency is $\theta^{l}$. For $q_{1}^{h}=1$ and $q_{1}^{l}=0$ in $t=1$, and $q_{2}^{h h}=q_{2}^{h l}=$ $q_{2}^{l l}=q_{2}^{l h}=\gamma$ in $t=2$, these two incentive compatibility conditions boil down to:

$$
W_{e}^{h}-W_{e}^{l} \geq 1-\theta^{h} \text { and } W_{e}^{h}-W_{e}^{l} \leq 1-\theta^{l}
$$

Given the aforementioned output schedules, the principal further has to respect the following participation constraints for the agent when his labor efficiency is $\theta^{h}$ and $\theta^{L}$ respectively:

$$
W_{e}^{h} \geq\left(1-\theta^{h}\right)(1+\gamma) \text { and } W_{e}^{l} \geq\left(1-\theta^{l}\right) \gamma
$$

It straightforwardly follows that $W_{e}^{l}=\left(1-\theta^{l}\right) \gamma$ and $W_{e}^{h}=\left(1-\theta^{l}\right) \gamma+\left(1-\theta^{h}\right)$ minimize expected wage transfers under the incentive compatibility and participation constraints. These wage transfers yield the principal the expected payoff $\Pi_{r p}^{C S}$ as stated in Lemma 8.

Proof of Proposition 5 We focus on the case $\mu \in\left(\hat{\mu}\left(\theta^{l}\right), \hat{\mu}\left(\theta^{h}\right)\right]$. According to Proposition 2 , it holds $\Pi^{D S}=\mu \theta^{h}+2 \mu\left(\theta^{h}+(1-\mu) \theta^{l}\right) \gamma$. Note that $\mu>\hat{\mu}\left(\theta^{l}\right)$ implies that $\mu \theta^{h}>\theta^{l}$ so that it follows

$$
\begin{aligned}
\Pi^{D S} & =\mu \theta^{h}+2 \mu\left(\theta^{h}+(1-\mu) \theta^{l}\right) \gamma \\
& =\mu \theta^{h}+\left(\mu \theta^{h}+\mu \theta^{h}+(1-\mu) \theta^{l}\right) \gamma \\
& >\mu \theta^{h}+\left(\theta^{l}+\mu \theta^{h}+(1-\mu) \theta^{l}\right) \gamma=\Pi_{r p}^{C S}
\end{aligned}
$$

Hence, $\Pi^{D S}>\Pi_{r p}^{C S}$. The principal's maximum payoff of a renegotiation-proof contract without early revelation coincides with $\Pi^{C P}$ as defined in Proposition 1 . The result then follows from Proposition 3 which shows $\Pi^{D S}>\Pi^{C P}$.

\section{Appendix B: Semi-Separating in the First Period}

We showed the optimality of delegation by focusing on pooling and full separation contracts. It is well known however that, in general, optimal contract may also involve semi-separation. This is due to the limited commitment of the principal, which leads to a failure of the revelation principle. In this appendix we show that the superiority of delegation is not due to our neglect of semi-separation. We, thereby, need to consider only semi-separating strategies under centralization, because we demonstrate that these outcomes lead to payoffs that are already lower than the principal's optimal contract under delegation with full separation. 
Bester and Strausz (2001) show that, despite imperfect commitment by the principal, direct mechanisms can implement any Pareto optimal outcome between the principal and the agent. These direct mechanisms induce the agent to report his type truthfully with a strictly positive probability, but may also require the agent to misreport with a strictly positive probability. In order to represent such mechanisms in our context, let $\alpha^{L} \in(0,1]$, where $L \in\{h, l\}$, denote the probability that the agent of type $\theta^{L}$ reports his type truthfully. A combination $\left(\alpha^{h}, \alpha^{l}\right)$ represents the agent's reporting strategy when facing a direct mechanism. Because we can always relabel messages, we may also restrict attention to reporting strategies with $\alpha^{h} \geq 1-\alpha^{l}$. Pooling contracts then coincide with the reporting strategy $\alpha^{h}=\alpha^{l}=1 / 2$ and (full) separation contracts coincide with the reporting strategy $\alpha^{h}=\alpha^{l}=1$. Because we focus in this appendix on reporting strategies that imply neither full pooling or full separation, we consider only reporting strategies from the set:

$$
\mathcal{A} \equiv\left\{\left(\alpha^{h}, \alpha^{l}\right) \in(0,1) \times(0,1) \mid \alpha^{h} \geq 1-\alpha^{l}\right\} \backslash\{(1 / 2,1 / 2),(1,1)\}
$$

Moreover, denote by $\mu_{2}^{L}$ the principal's posterior belief that the agent is efficient after he reports about $\theta^{L}$ in $t=1$. For a given reporting strategy $\alpha^{h}$ and $\alpha^{l}$, the principal's beliefs $\mu_{2}^{h}$ and $\mu_{2}^{l}$, in equilibrium, must satisfy Bayes' rule:

$$
\mu_{2}^{h}\left(\alpha^{h}, \alpha^{l}\right) \equiv \frac{\mu \alpha^{h}}{\mu \alpha^{h}+(1-\mu) \alpha^{l}} \quad \text { and } \quad \mu_{2}^{l}\left(\alpha^{h}, \alpha^{l}\right) \equiv \frac{\mu\left(1-\alpha^{h}\right)}{\mu\left(1-\alpha^{h}\right)+(1-\mu) \alpha^{l}} .
$$

For any reporting strategy $\left(\alpha^{h}, \alpha^{l}\right) \in \mathcal{A}$ expression (B1) implies $\mu_{2}^{l}<\mu<\mu_{2}^{h}$.

In an equilibrium, the principal's offer in $t=2$ is sequentially rational given the agent's reporting behavior. We can, therefore, view it as contingent on the agent's report on $\theta^{L}$ and also on the principal's observation of $\theta^{K}$. The crucial question is whether the sequentially rational contract itself is a pooling or a separating one. Thus, let $\rho^{L K}$ denote the probability that the principal offers a pooling contract in $t=2$ given the agent's report on $\theta^{L}$ and her observation of $\theta^{K}$. By Lemma 1 , the principal's sequentially rational behavior implies:

$$
\rho^{L K} \in \begin{cases}1 & \text { if } \mu_{2}^{L}<\hat{\mu}\left(\theta^{K}\right) \\ {[0,1]} & \text { if } \mu_{2}^{L}=\hat{\mu}\left(\theta^{K}\right) \\ 0 & \text { if } \mu_{2}^{L}>\hat{\mu}\left(\theta^{K}\right)\end{cases}
$$

From $\mu_{2}^{l}<\mu<\mu_{2}^{h}$, it then follows that $\rho^{l K} \geq \rho^{h K}$. This means that, in equilibrium, it is more likely that the principal offers a pooling contract if the agent reports that $\theta^{L}=\theta^{l}$. It 
follows, because the subsequent decrease in the principal's belief that the agent is efficient shifts the trade-off between a pooling and a separating contract more in favor of the pooling contract.

On the equilibrium path, any sequentially rational contract in $t=2$, leaves a rent to the agent only if the contract is a pooling one and, in this case, the rent is $\Delta \theta \gamma$. The agent of type $\theta^{h}$ therefore expects to receive the rent with probability $\mu \rho^{L h}+(1-\mu) \rho^{L l}$ when he reports $\theta^{L}$. Because, in equilibrium, the agent's reporting strategy $\left(\alpha^{h}, \alpha^{l}\right)$ must be optimal, the reporting probability $\alpha^{h}$ satisfies:

$$
\begin{aligned}
& \alpha^{h} \in \arg \max _{\hat{\alpha}^{h}} \hat{\alpha}^{h}\left[T_{1}^{h}-\left(1-\theta^{h}\right)+\left(\mu \rho^{h h}+(1-\mu) \rho^{h l}\right) \Delta \theta \gamma\right] \\
& +\left(1-\hat{\alpha}^{h}\right)\left[T_{1}^{l}+\left(\mu \rho^{l h}+(1-\mu) \rho^{l l}\right) \Delta \theta \gamma\right] .
\end{aligned}
$$

Similarly, because the agent of type $\theta^{l}$ will not receive a rent in $t=2$, the reporting strategy $\alpha^{l}$, in equilibrium, satisfies:

$$
\alpha^{l} \in \arg \max _{\hat{\alpha}^{l}} \hat{\alpha}^{l} T_{1}^{l}+\left(1-\hat{\alpha}^{l}\right)\left[T_{1}^{h}-\left(1-\theta^{l}\right)\right]
$$

With the help of $\alpha^{h}, \alpha^{l}$ and $\rho^{L K}$, the principal's expected payoff in the beginning of $t=1$ can be written as:

$$
\begin{aligned}
\Pi_{\text {semi }}^{C}= & \mu\left\{\alpha^{h}\left[1-T_{1}^{h}+\left(\theta^{h}+\widetilde{\theta}-\left(\mu \rho^{h h}+(1-\mu) \rho^{h l}\right) \Delta \theta\right) \gamma\right]\right. \\
& \left.+\left(1-\alpha^{h}\right)\left[-T_{1}^{l}+\left(\theta^{h}+\widetilde{\theta}-\left(\mu \rho^{l h}+(1-\mu) \rho^{l l}\right) \Delta \theta\right) \gamma\right]\right\} \\
& +(1-\mu)\left\{\alpha^{l}\left[-T_{1}^{l}+\left(\mu \rho^{l h}\left(\theta^{l}+\theta^{h}\right)+(1-\mu) \rho^{l l}\left(\theta^{l}+\theta^{l}\right)\right) \gamma\right]\right. \\
& \left.+\left(1-\alpha^{l}\right)\left[1-T_{1}^{h}+\left(\mu \rho^{h h}\left(\theta^{l}+\theta^{h}\right)+(1-\mu) \rho^{h l}\left(\theta^{l}+\theta^{l}\right)\right) \gamma\right]\right\} .
\end{aligned}
$$

Therefore, if semi-separation under centralization is optimal, then there exists a combination $\left(\alpha^{h}, \alpha^{l}\right) \in \mathcal{A}$ that maximizes $\Pi_{\text {semi }}^{C}$ subject to (B1), (B2), (B3), and (B4). In order to solve this problem, we first show that, with semi-separation, optimal reporting strategies are necessarily such that they induce a belief of the principal that makes her exactly indifferent about offering a pooling or separating contract in $t=2$. The intuition behind this result is that these beliefs bring the principal's myopic second period behavior in line with the principal's ex ante perspective of $t=1$. Because, in equilibrium, the principal's beliefs depend on the agent's reporting strategy, his reporting behavior is a key instrument for controlling the principal's myopic behavior in $t=2$. 
Lemma 9 If reporting behaviors in $\mathcal{A}$ are optimal, then there exist an optimal $\alpha^{h}$ and $\alpha^{l}$ such that $i) \alpha^{h}=1$ and $\mu_{2}^{h}\left(1, \alpha^{l}\right)=\hat{\mu}\left(\theta^{h}\right)$, or $\left.i i\right) \alpha^{l}=1$ and $\mu_{2}^{l}\left(\alpha^{h}, 1\right)=\hat{\mu}\left(\theta^{l}\right)$, or $\left.i i i\right)$ $\mu_{2}^{h}\left(\alpha^{h}, \alpha^{l}\right)=\hat{\mu}\left(\theta^{h}\right)$ and $\mu_{2}^{l}\left(\alpha^{h}, \alpha^{l}\right)=\hat{\mu}\left(\theta^{l}\right)$.

Proof. Suppose $\alpha^{h}$ and $\alpha^{l}$ are optimal and stochastic and $\mu_{2}^{h}\left(\alpha^{h}, \alpha^{l}\right) \neq \hat{\mu}\left(\theta^{h}\right)$ and $\mu_{2}^{l}\left(\alpha^{h}, \alpha^{l}\right) \neq \hat{\mu}\left(\theta^{l}\right)$. Because $\rho^{L K}$ only changes at $\hat{\mu}\left(\theta^{L}\right)$, (B5) is linear in $\alpha^{h}$ and $\alpha^{l}$ (for sufficiently small changes in $\alpha^{h}$ and $\alpha^{l}$ ). However, the optimality of $\alpha^{h}$ and $\alpha^{l}$ implies that (B5) can neither be increasing nor decreasing in $\alpha^{h}$ or $\alpha^{l}$. Thus, (B5) must be independent of $\alpha^{h}$ and $\alpha^{l}$ and we can increase or decrease $\alpha^{h}$ and $\alpha^{l}$ so that $\mu_{2}^{h}\left(\alpha^{h}, \alpha^{l}\right)=\hat{\mu}\left(\theta^{h}\right)$ or $\mu_{2}^{l}\left(\alpha^{h}, \alpha^{l}\right)=\hat{\mu}\left(\theta^{l}\right)$. Because the original $\rho^{L K}$ is consistent with these adapted $\alpha^{h}$ and $\alpha^{l}$, it yields the same expected payoff to the principal and is hence also optimal. Suppose now there exist no deterministic reporting strategy at the optimum. Then, $\alpha^{h} \in(0,1)$ and $\alpha^{l} \in(0,1)$ so that $\mu_{2}^{h}\left(\alpha^{h}, \alpha^{l}\right)=\hat{\mu}\left(\theta^{h}\right)$ or $\mu_{2}^{l}\left(\alpha^{h}, \alpha^{l}\right)=\hat{\mu}\left(\theta^{l}\right)$. If $\mu_{2}^{h}\left(\alpha^{h}, \alpha^{l}\right)=\hat{\mu}\left(\theta^{h}\right)$ and $\mu_{2}^{l}\left(\alpha^{h}, \alpha^{l}\right) \neq \hat{\mu}\left(\theta^{l}\right)$, then we can specify the implicit function $\widetilde{\alpha}^{l}\left(\alpha^{h}\right)$ by $\mu_{2}^{h}\left(\alpha^{h}, \widetilde{\alpha}^{l}\left(\alpha^{h}\right)\right)=\mu_{2}^{h}\left(\alpha^{h}, \alpha^{l}\right)$. Because $\widetilde{\alpha}^{l}$ is necessarily linear in $\alpha^{h}$ and (B5) is linear in both $\alpha^{h}$ and $\alpha^{l}$, replacing $\alpha_{l}$ with $\widetilde{\alpha}^{l}\left(\alpha^{h}\right)$ in (B5) yields an expression for the principal's payoff $\widetilde{\Pi}_{\text {semi }}^{C}\left(\alpha^{h}\right)$ that is linear in $\alpha^{h}$ for a small change in $\alpha^{h}$. Now, suppose $\mu_{2}^{l}\left(\alpha^{h}, \alpha^{l}\right)<\hat{\mu}\left(\theta^{l}\right)$, and consider the interval $\left[0, \bar{\alpha}^{h}\right]$, where $\bar{\alpha}^{h}$ is such that $\mu_{2}^{l}\left(\bar{\alpha}^{h}, \widetilde{\alpha}^{l}\left(\bar{\alpha}^{h}\right)\right)=\hat{\mu}\left(\theta^{l}\right)$. Note that if $\alpha^{h}$ and $\alpha^{l}$ maximize (B5), then they must maximize $\widetilde{\Pi}_{\text {semi }}^{C}\left(\alpha^{h}\right)$ over $\left[0, \bar{\alpha}^{h}\right]$ at $\alpha^{h}$, because $\rho^{L K}$ remains unchanged for any combination $\left\{\bar{\alpha}^{h}, \alpha^{h}, \widetilde{\alpha}^{l}\left(\alpha^{h}\right)\right\}$ with $\alpha^{h} \in\left[0, \bar{\alpha}^{h}\right]$. However, because $\widetilde{\Pi}_{\text {semi }}^{C}\left(\alpha^{h}\right)$ is linear in $\alpha^{h}$, it must be maximized at either 0 or $\bar{\alpha}^{h}$. It follows that (B5) must also be optimized for $\left\{\alpha^{h}, \alpha^{l}\right\}=\left\{0, \widetilde{\alpha}^{l}(0)\right\}$ or $\left\{\alpha^{h}, \alpha^{l}\right\}=\left\{\bar{\alpha}^{h}, \widetilde{\alpha}^{l}\left(\bar{\alpha}^{h}\right)\right\}$. The first case, $\left\{\alpha^{h}, \alpha^{l}\right\}=\left\{0, \widetilde{\alpha}^{l}(0)\right\}$, contradicts the assumption that there only exist optimal outcome in purely mixed strategy, implying that $\left\{\bar{\alpha}^{h}, \widetilde{\alpha}^{l}\left(\bar{\alpha}^{h}\right)\right\}$ must be optimal. Yet, as we needed to show, this implies $\mu_{2}^{l}=$ $\hat{\mu}\left(\theta^{l}\right)$. If $\mu_{2}^{l}\left(\alpha^{h}, \alpha^{l}\right)>\hat{\mu}\left(\theta^{l}\right)$ similar arguments show that there must also exist optimal $\alpha^{h}$ and $\alpha^{l}$ with $\mu_{2}^{h}\left(\alpha^{h}, \alpha^{l}\right)=\hat{\mu}\left(\theta^{h}\right)$ and $\mu_{2}^{l}\left(\alpha^{h}, \alpha^{l}\right)=\hat{\mu}\left(\theta^{l}\right)$. The case of $\mu_{2}^{h}\left(\alpha^{h}, \alpha^{l}\right) \neq \hat{\mu}\left(\theta^{h}\right)$ and $\mu_{2}^{l}\left(\alpha^{h}, \alpha^{l}\right)=\hat{\mu}\left(\theta^{l}\right)$ can be treated similarly to show that there must then also exist optimal $\alpha^{h}$ and $\alpha^{l}$ with $\mu_{2}^{h}\left(\alpha^{h}, \alpha^{l}\right)=\hat{\mu}\left(\theta^{h}\right)$ and $\mu_{2}^{l}\left(\alpha^{h}, \alpha^{l}\right)=\hat{\mu}\left(\theta^{l}\right)$.

With this characterization of reporting behavior, we may compute the principal's maximum payoff for the three different cases and compute her maximum payoff, $\Pi_{\text {semi }}^{C}$.

Proposition 6 The principal's payoff under delegation exceeds her payoff under centralization that induces a semi-separating equilibrium for $\mu<\frac{2 \theta^{h} \theta^{l}}{\left(\theta^{h}+\theta^{l}\right) \Delta \theta \gamma}$ and, in particular, for any $\mu<\hat{\mu}\left(\theta^{h}\right)$. 
Proof. We compute the payoffs for the three cases in Lemma 9. First, suppose $\alpha^{h}=1$ and $\mu_{2}^{h}\left(1, \alpha^{l}\right)=\hat{\mu}\left(\theta^{h}\right)$ is optimal. Because $\mu_{2}^{h}>\mu$, this case exists only if $\hat{\mu}\left(\theta^{h}\right)>\mu$. Moreover, it holds $\mu_{2}^{l}=0$ so that $\rho^{l h}=\rho^{l l}=1$ and, due to $\mu_{2}^{h}\left(1, \alpha^{l}\right)=\hat{\mu}\left(\theta^{h}\right)>\hat{\mu}\left(\theta^{l}\right)$ it follows $\rho^{h l}=0$ and the principal is indifferent between pooling and separation, and thus her expected payoff is independent of $\rho^{h h}$. Expression (B5), therefore, simplifies to:

$$
\begin{aligned}
& \mu\left[1-T_{1}^{h}+\left(\theta^{h}+\tilde{\theta}\right) \gamma\right] \\
& +(1-\mu)\left\{\alpha^{l}\left[-T_{1}^{l}+\left(\mu\left(\theta^{l}+\theta^{h}\right)+(1-\mu)\left(\theta^{l}+\theta^{l}\right)\right) \gamma\right]+\left(1-\alpha^{l}\right)\left[1-T_{1}^{h}\right]\right\},
\end{aligned}
$$

and is to maximize under the incentive constraints,

$$
T_{1}^{h} \geq\left(1-\theta^{h}\right)+\left(1-\mu \rho^{h h}\right) \Delta \theta \gamma+T_{1}^{l} \quad \text { and } \quad T_{1}^{l}=T_{1}^{h}-\left(1-\theta^{l}\right),
$$

and the participation constraints,

$$
T_{1}^{l} \geq 0 \quad \text { and } \quad T_{1}^{h} \geq 1-\theta^{h}-\mu \rho^{h h} \Delta \theta \gamma .
$$

Because $\rho^{h h}$ does not influence the principal's expected payoff, but the constraints are unambiguously relaxed for larger $\rho^{h h}, \rho^{h h}=1$ is optimal. As usual, the participation constraint for type $\theta^{l}$ together with the incentive constraint for type $\theta^{h}$ imply the participation constraint for type $\theta^{h}$. It also follows that the two incentive constraints are consistent only if $(1-\mu) \gamma \leq 1$. In this case, $T_{1}^{l}=T_{1}^{h}-\left(1-\theta^{l}\right)$ implies that the incentive constraint for type $\theta^{h}$ is satisfied, because it follows:

$$
\left(1-\theta^{h}\right)+(1-\mu) \Delta \theta \gamma+T_{1}^{l}=(1-\mu) \Delta \theta \gamma-\Delta \theta+T_{1}^{h} \leq T_{1}^{h} .
$$

Hence, an optimal solution exists only if $(1-\mu) \gamma \leq 1$ and it exhibits:

$$
T_{1}^{h}=1-\theta^{l} \quad \text { and } \quad T_{1}^{l}=0,
$$

with the payoff:

$$
\Pi_{\text {semi }}^{C}=\frac{2 \mu \theta^{h} \theta^{l}+\left[\left(2 \mu \theta^{h}-\mu^{2} \Delta \theta\right) \Delta \theta+2 \theta^{l}\left(\theta^{h}+\theta^{l}\right)\right] \gamma}{\theta^{h}+\theta^{l}},
$$

which is smaller than $\Pi^{D S}$ for the entire implementable range $\mu<\hat{\mu}\left(\theta^{h}\right)$.

Suppose now $\alpha^{l}=1$ and $\mu_{2}^{l}\left(\alpha^{h}, 1\right)=\hat{\mu}\left(\theta^{l}\right)$ is optimal. Because $\mu_{2}^{l}<\mu$, this case exists only if $\hat{\mu}\left(\theta^{l}\right)<\mu$. Moreover, it holds $\mu_{2}^{h}=1$ so that $\rho^{h h}=\rho^{h l}=0$ and, due to 
$\mu_{2}^{l}\left(\alpha^{h}, 1\right)=\hat{\mu}\left(\theta^{l}\right)<\hat{\mu}\left(\theta^{h}\right)$ it follows $\rho^{l h}=1$ and the principal is indifferent between separation and pooling. Hence the principal's payoff in (B5) is independent of $\rho^{l l}$, and simplifies to:

$$
\begin{aligned}
& \mu\left\{\alpha^{h}\left[1-T_{1}^{h}+\left(\theta^{h}+\tilde{\theta}\right) \gamma\right]+\left(1-\alpha^{h}\right)\left[-T_{1}^{l}+\left(\theta^{h}+\widetilde{\theta}-\mu \Delta \theta\right) \gamma\right]\right\} \\
& +(1-\mu)\left[-T_{1}^{l}+\mu\left(\theta^{l}+\theta^{h}\right) \gamma\right]
\end{aligned}
$$

and is to maximize under the incentive constraints,

$$
T_{1}^{h}=\left(1-\theta^{h}\right)+\left(\mu+(1-\mu) \rho^{l l}\right) \Delta \theta \gamma+T_{1}^{l} \quad \text { and } \quad T_{1}^{l} \geq T_{1}^{h}-\left(1-\theta^{l}\right),
$$

and the participation constraints,

$$
T_{1}^{l} \geq 0 \quad \text { and } \quad T_{1}^{h} \geq 1-\theta^{h}
$$

Again, the participation constraint of $\theta^{h}$ is implied by the incentive constraint of $\theta^{h}$ and the participation constraint of $\theta^{l}$. Moreover, because the principal's expected payoff is decreasing in $T_{1}^{h}$, and $T_{1}^{h}$ itself is decreasing in $\rho^{l l}$, a smaller $T_{1}^{h}$ relaxes the incentive constraint for type $\theta^{l}$, and it follows that $\rho^{l l}=0$ must be optimal. It then follows that the two incentive constraints are consistent only if $\mu \gamma \leq 1$. Only in this case, an optimal solution exists:

$$
T_{1}^{h}=\left(1-\theta^{h}\right)+\mu \Delta \theta \gamma \quad \text { and } \quad T_{1}^{l}=0,
$$

with the payoff:

$$
\Pi_{\text {semi }}^{C}=\frac{\mu \theta^{h^{2}}-(2-\mu) \theta^{h} \theta^{l}+\mu(2-\mu)\left(\theta^{h^{2}}-\theta^{l^{2}}\right) \gamma}{\Delta \theta} .
$$

This is smaller than $\Pi^{D S}$ for the range $\mu \in\left(\hat{\mu}\left(\theta^{l}\right), \hat{\mu}\left(\theta^{h}\right)\right]$. For $\mu>\hat{\mu}\left(\theta^{h}\right)$, straightforward calculations show that $\Pi_{\text {semi }}^{C}<\Pi^{D S}$ when

$$
\mu<\frac{2 \theta^{h} \theta^{l}}{\left(\theta^{h}+\theta^{l}\right) \Delta \theta \gamma} .
$$

Finally, suppose $\mu_{2}^{h}\left(\alpha^{h}, \alpha^{l}\right)=\hat{\mu}\left(\theta^{h}\right)$ and $\mu_{2}^{l}\left(\alpha^{h}, \alpha^{l}\right)=\hat{\mu}\left(\theta^{l}\right)$ is optimal. Because $\mu_{2}^{l}<\mu<$ $\mu_{2}^{h}$ this case exists only if $\hat{\mu}\left(\theta^{l}\right)<\mu<\hat{\mu}\left(\theta^{h}\right)$. Due to $\mu_{2}^{h}\left(\alpha^{h}, \alpha^{l}\right)=\hat{\mu}\left(\theta^{h}\right)>\hat{\mu}\left(\theta^{l}\right)$, it follows $\rho^{h l}=0$ and, due to $\mu_{2}^{l}\left(\alpha^{h}, \alpha^{l}\right)=\hat{\mu}\left(\theta^{l}\right)<\hat{\mu}\left(\theta^{h}\right)$, it follows $\rho^{l h}=1$. Moreover, the principal is indifferent to $\rho^{h h}$ and $\rho^{l l}$, and hence her expected payoff must be independent of both $\rho^{h h}$ and $\rho^{l l}$. The expression in (B5) simplifies to:

$$
\begin{aligned}
& \mu\left\{\alpha^{h}\left[1-T_{1}^{h}+\left(\theta^{h}+\widetilde{\theta}\right) \gamma\right]+\left(1-\alpha^{h}\right)\left[-T_{1}^{l}+\left(\theta^{h}+\widetilde{\theta}-\mu \Delta \theta\right) \gamma\right]\right\} \\
& +(1-\mu)\left\{\alpha^{l}\left[-T_{1}^{l}+\mu\left(\theta^{l}+\theta^{h}\right) \gamma\right]+\left(1-\alpha^{l}\right)\left[1-T_{1}^{h}\right]\right\}
\end{aligned}
$$


and is to maximize under the incentive constraints,

$$
T_{1}^{h}=\left(1-\theta^{h}\right)+\left(\mu\left(1-\rho^{h h}\right)+(1-\mu) \rho^{l l}\right) \Delta \theta \gamma+T_{1}^{l} \quad \text { and } \quad T_{1}^{l}=T_{1}^{h}-\left(1-\theta^{l}\right),
$$

and the participation constraints,

$$
T_{1}^{l} \geq 0 \quad \text { and } \quad T_{1}^{h} \geq 1-\theta^{h}+\mu \rho^{h h} \Delta \theta \gamma
$$

The incentive constraints are consistent only if $\mu\left(1-\rho^{h h}\right)+(1-\mu) \rho^{l l}=1 / \gamma$. This implies $T_{1}^{h}=1-\theta^{l}+T_{1}^{l}$. Substituting out the transfer $T_{1}^{h}$ in (B6) demonstrates that the expression is decreasing in $T_{1}^{l}$. The optimal transfer is $T_{1}^{l}=0$, which satisfies the participation constraint of type $\theta^{h}$ for $\rho^{h h}=0$. It yields the principal the payoff:

$$
\Pi_{\text {semi }}^{C}=\frac{2 \theta^{h} \theta^{l}\left(\mu\left(\theta^{h}+\theta^{l}\right)-2 \theta^{l}\right)+\mu(2-\mu)\left(\theta^{h}+\theta^{l}\right) \Delta \theta^{2} \gamma}{\Delta \theta^{2}}
$$

which is smaller than $\Pi^{D S}$ for the entire implementable range $\mu \in\left(\hat{\mu}\left(\theta^{l}\right), \hat{\mu}\left(\theta^{h}\right)\right)$.

For $\mu \in\left(\hat{\mu}(0), \hat{\mu}\left(\theta^{h}\right)\right)$, delegation is optimal even when considering semi-separating strategies. For $\mu>\hat{\mu}\left(\theta^{h}\right)$, the region of delegation in Figure 1 survives unless $\mu$ is very high. With the semi-separating strategy, the probability of no production in both periods is strictly positive. However, there is a chance that separation takes places in $t=1$ with no rent provision in $t=2$. Thus, if it is highly likely that the agent is efficient, the principal prefers to take a risk in $t=1$ by inducing the agent's randomization. By doing so, the principal's cost to separate the agent's types in $t=1$ becomes lower. If, however, the agent is not highly likely to be efficient, the principal is better off by avoiding the semi-separating strategy. 


\section{References}

[1] Acemoglu, D., Aghion, P., Lelarge, C., Van Reenen, J. and Zilibotti, F. (2007), "Technology, Information, and the Decentralization of the Firm," Quarterly Journal of Economics, 4, 1759 - 1799.

[2] Aghion, P. and Tirole, J. (1997), "Formal and Real Authority in Organizations," Journal of Political Economy, 105, 1 - 29.

[3] Aghion, P., Dewatripont, M. and Rey, P. (2004), "Transferable Control," Journal of the European Economic Association, 2, 115 - 138.

[4] Alonso, R., Dessein, W. and Matouschek, N. (2008), "When Does Coordination Require Centralization?," American Economic Review, 98, 145 - 179.

[5] Argyris, C. (1982), "The Executive Mind and Double-Loop Learning," Organizational Dynamics, 11, 5 - 22 .

[6] Baron, D. and Besanko, D. (1992), "Information, Control, and Organizational Structure," Journal of Economics and Management Strategy, 1, 237 - 275.

[7] Beaudry, P. and Poitevin, M. (1995), "Contract Renegotiation: A Simple Framework and Implications for Organization Theory," Canadian Journal of Economics, 28, $302-335$.

[8] Bester, H. and Strausz, R. (2001), "Contracting with Imperfect Commitment and the Revelation Principle: The Single Agent Case," Econometrica, 69, 1077 - 1098.

[9] Bolton, P. and Dewatripont, M. (2005), Contract Theory, MIT Press, Cambridge, MA.

[10] Bolton, P. and Scharfstein, D. (1990), "A Theory of Predation Based on Agency Problems in Financial Contracting," American Economic Review, 80, 93 - 106.

[11] Chen, B. (2010), "Samsung Emerges as a Potent Rival to Apple's Cool," The New York Times, February 10.

[12] Coase, R. (1937), "The Nature of the Firm," Economica, 4, 386 - 405. 
[13] Crémer, J. (1995), "Arm's Length Relationships," Quarterly Journal of Economics, $110,275-295$.

[14] Cyert, R. and March, J. (1963/1992), A Behavioral Theory of the Firm, Blackwell Publishers, Malden, MA

[15] Dequiedt, V. and Martimort, D. (2004), “Delegated Monitoring versus Arm'sLength Contracting," International Journal of Industrial Organization, 22, 951 - 981.

[16] Dessein, W. (2002), "Authority and Communication in Organizations," Review of Economic Studies, 69, 811 - 838.

[17] Dewatripont, M. and Maskin, E. (1995), "Contractual Contingencies and Renegotiation," Rand Journal of Economics, 26, 704 - 719.

[18] Drugov, M. (2010), "Information and Delay in an Agency Model," Rand Journal of Economics, 41, 598 - 615.

[19] Editorial - Economist (2000), "How Mergers Go Wrong," Economist, July 20 th.

[20] Hirao, Y. (1993), "Task Assignment and Agency Structures," Journal of Economics and Management Strategy, 2, 299 - 323.

[21] Holmström, B. and Milgrom, P. (1994), "The Firm as an Incentive System," American Economic Review, 84, 972 - 991.

[22] Kastl, J., Martimort, D. and Piccolo, S. (2013), "Delegation, Ownership Concentration and R\&D Spending: Evidence from Italy," Journal of Industrial Economics, $61,84-107$.

[23] Laffont, J.-J. and Martimort, D. (1998), "Collusion and Delegation," Rand Journal of Economics, 29, 280 - 305.

[24] Laffont, J.-J. and Tirole, J. (1987), "Comparative Statics of the Optimal Dynamic Incentive Contract," European Economic Review, 31, 901 - 926.

[25] Laffont, J.-J. and Tirole, J. (1988), "The Dynamics of Incentive Contracts," Econometrica, 56, 1153 - 1175. 
[26] Laffont, J.-J. and Tirole, J. (1993), A Theory of Incentives in Procurement and Regulation, MIT Press, Cambridge, MA.

[27] Lashinsky, A. (2012), Inside Apple: How America's Most Admired-and SecretiveCompany Really Works, John Murray Publisher, London, UK.

[28] Maskin, E. and Tirole, J. (1990) "The Principal-Agent Relationship with an Informed Principal: The Case of Private Values," Econometrica, 58, 379-409

[29] Martimort, D. (1999), "Renegotiation Design with Multiple Regulators," Journal of Economic Theory, 88, 261 - 293.

[30] Meyer, M., Olsen, T. and Torsvik, G. (1996), "Limited Intertemporal Commitment and Job Design," Journal of Economic Behavior and Organization, 31, 401 - 417.

[31] Michell, A. (2010), Samsung Electronics and the Struggle for Leadership of the Electronics Industry, Wiley, Hoboken, NJ.

[32] Mintzberg, H. (1979), The Structuring of Organizations, Prentice-Hall, Englewood Cliffs, NJ.

[33] Olsen, T. and Torsvik, G. (1993), "The Ratchet Effect in Common Agency: Implications for Regulation and Privatization," Journal of Law, Economics and Organization, $9,136-158$.

[34] Olsen, T. and Torsvik, G. (1995), "Intertemporal Common Agency and Organizational Design: How Much Decentralization?," European Economic Review, 39, 1405 1428.

[35] Olsen, T. (1996), "Agency Costs and the Limits of Integration," Rand Journal of Economics, 27, 479 - 501.

[36] Olsen, T. and Torsvik, G. (2000), "Discretion and Incentives in Organizations," Journal of Labor Economics, 18, 377 - 404.

[37] Poitevin, M. (2000), "Can the Theory of Incentives Explain Decentralization?," Canadian Journal of Economics, 33, 878 -906. 
[38] Rajan, R. and Zingales, L. (2001), "The Firm as a Dedicated Hierarchy: A Theory of The Origins and Growth of Firms," Quarterly Journal of Economics, 116, 805 - 851.

[39] Riordan, M. (1990), "What is Vertical Integration?," in Firm as a Nexus of Treaties by M. Aoki, B. Gustafsson, O. Williamson (eds.) London, 94-111.

[40] Roberts, J. (2004), The Modern Firm: Organizational Design for Performance and Growth, Oxford University Press, Oxford, UK.

[41] Schein, E. (2004), Organizational Culture and Leadership, Weily: Jossy-Bass, Hoboken, NJ.

[42] Severinov, S. (2008), "The Value of Information and Optimal Organization," Rand Journal of Economics, 39, 238 - 265.

[43] Williamson, O. (1985), The Economic Institutions of Capitalism, The Free Press, New York, NY. 


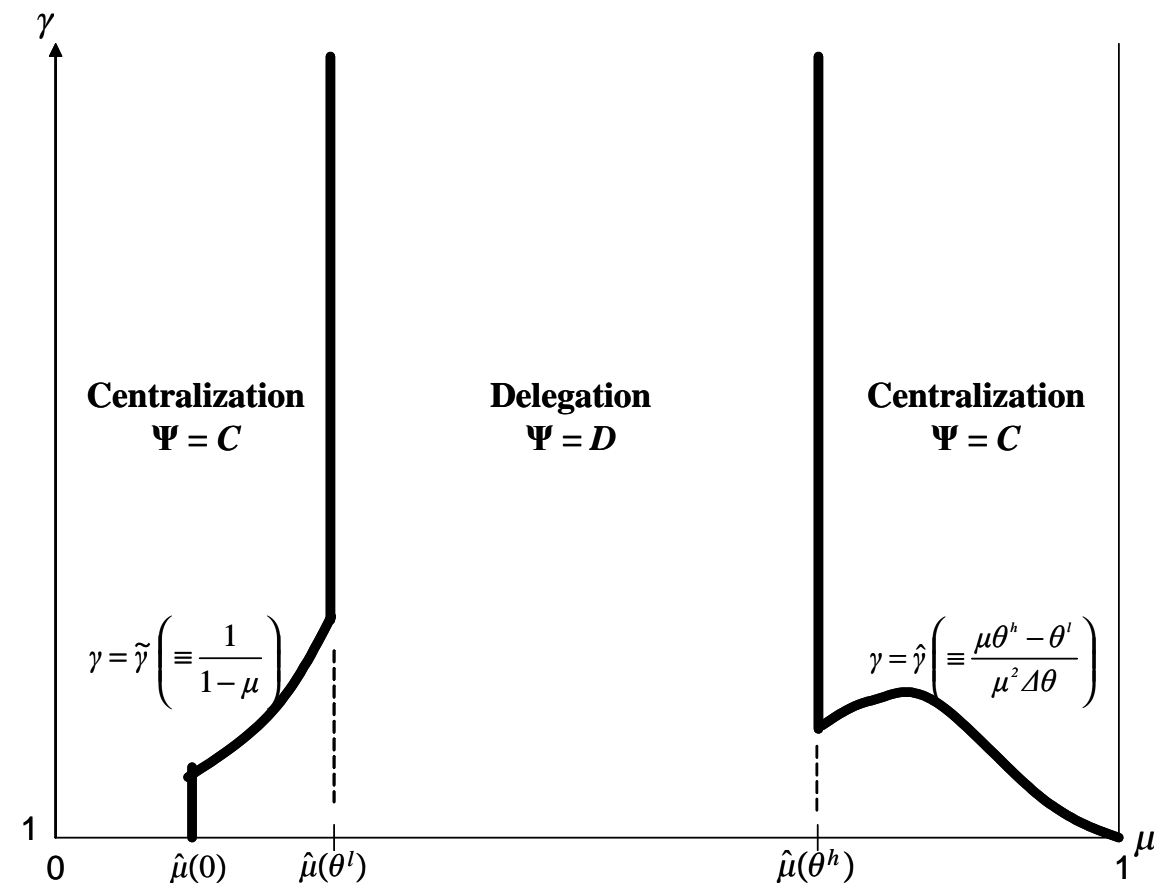

Fig 1. Centralization versus Delegation 